\title{
Measurement error and deconvolution in spaces of generalized functions
}

\author{
Victoria Zinde-Walsh* \\ McGill University and CIREQ \\ victoria.zinde-walsh@mcgill.ca
}

(514) 3984834

June 3, 2022

\begin{abstract}
*The support of the Social Sciences and Humanities Research Council of Canada (SSHRC), the Fonds québecois de la recherche sur la société et la culture (FRQSC) is gratefully acknowledged. P.C.B.Phillips provided invaluable encouragement, comments and advice; I also thank J. Galbraith for his support and comments. The Associate Editor, Y. Kitamura and anonymous referees provided corrections, comments and suggestions that were extremely helpful; I am very grateful to them.
\end{abstract}


Running head: Deconvolution in generalized functions

Victoria Zinde-Walsh

Department of Economics, McGill University

855 Sherbrooke Street West,

Montreal, Quebec, Canada

H3A 2T7

\section{Abstract}

This paper considers convolution equations that arise from problems such as measurement error and non-parametric regression with errors in variables with independence conditions. The equations are examined in spaces of generalized functions to account for possible singularities; this makes it possible to consider densities for arbitrary and not only absolutely continuous distributions, and to operate with Fourier transforms for polynomially growing regression functions. Results are derived for identification and well-posedness in the topology of generalized functions for the deconvolution problem and for some regression models. Conditions for consistency of plug-in estimation for these models are derived. 


\section{Introduction}

The focus of this paper is on convolution equations arising in models with measurement error. Reviews of measurement error models are in Carroll and Stefanski (2006), Chen, Hong and Nekipelov (2011), Meister(2009). The convolution equations that are examined here also arise in other contexts; various models that go beyond measurement error models are enumerated in Zinde-Walsh (2012). This paper is devoted to the mathematical treatment of such equations.

Start with the classical measurement error where the variable of interest $x^{*}$ is observed with error, $u$ :

$$
z=x^{*}+u
$$

Here the density of $x^{*}$ is the function of interest, denoted $g$; the observed $z$ has density $w$; suppose that the measurement/contamination error $u$ is independent of $x^{*}$ and has density $f$. Then the convolution equation

$$
g * f=w,
$$

holds. When densities exist, $g * f$ denotes $\int g(z-u) f(u) d u$. In problems that often arise in image processing, epidemiology, medicine the error density $f$ could be assumed known, e.g. the error is Gaussian noise.

The assumption of known error distribution may not be realistic; if $f$ is 
not known additional conditions are needed to identify $g$. If another observation, $x$, on $x^{*}$ is available: $x=x^{*}+u_{x}$ conditions under which a unique solution exists for the density of interest, $g$, were given by Kotlyarski (1967) in the case of independence between $x^{*}$ and $u_{x}$; the approach there was to consider the joint characteristic function of the two measurements.

The assumption of independence of the error in the second measurement may be too strong for some applications, for example in Cunha, Heckman and Schennach (2010) one measurement for the latent variable representing a skill of a child was constructed from test scores where one could plausibly assume independence for measurement error, but the extra measurements came from reports by teachers and parents where such an assumption could be unrealistic. Assume that for the error of the second measurement, $u_{x}$, the conditional expectation is zero: $E\left(u_{x} \mid x^{*}, u\right)=0$ (but heteroscedasticity is not ruled out). Denote by $x_{k}$ the $k-t h$ component of $x=\left(x_{1}, \ldots, x_{d}\right)$ and by $h_{k}(x)$ the function $x_{k} g(x)$, and assume existence of density weighted conditional moments $w_{2 k}(z)=E\left(w(z) x_{k} \mid z\right)$. Then in addition to the equation (2) more convolution equations can be written:

$$
h_{k} * f=w_{2 k}, k=1, \ldots, d
$$

Indeed (with integration here and everywhere in this paper over the whole 
space),

$$
E\left(w(z) x_{k} \mid z\right)=E\left(w(z) x_{k}^{*} \mid z\right)=\int\left(z_{k}-u_{k}\right) g(z-u) f(u) d u
$$

Therefore a system of convolution equations arises for the unknown function $g$ and functions $h_{k}$, where $h_{k}(x)=x_{k} g(x)$,

$$
\begin{aligned}
g * f & =w_{1} \\
\left(h_{k}\right) * f & =w_{2 k}, k=1, \ldots d .
\end{aligned}
$$

Another model that leads to a system of equations is nonparametric regression with Berkson error (see, e.g. Meister, 2009 for review). Consider a nonparametric regression model

$$
y=g(x)+u_{y}
$$

where $x$ may be correlated with the error, $u_{y}$, but where some instruments, $z$, are available such that

$$
z=x+u
$$

with $z$ is independent of $u$ (Berkson error) and $E\left(u_{y} \mid z\right)=0$. Denote the density of $x$ by $f_{x}$, density of $z$ by $f_{z}$, density of $u$ by $f_{u}$ and correspondingly 
that of $-u$ by $f_{-u}$. Then equation (4) gives

$$
f_{x}=f_{z} * f_{-u}
$$

Additionally if expectation conditional on $z$ exists, using independence between $z$ and $u$,

$$
w(z)=E(y \mid z)=E(g(x) \mid z)=\int g(z-u) f_{u}(u) d u
$$

then

$$
g * f_{u}=w
$$

The system of equations (5,6) involves two unknown functions, $f_{u}$ and $g$, where usually the interest is in the regression function.

The regression function could have instead of an observable argument, $x$, a mismeasured or latent argument, $x^{*}$. The model could provide more equations if another measurement, $x$, on $x^{*}$ were available. Consider

$$
\begin{aligned}
& y=g\left(x^{*}\right)+u_{y} ; \\
& x=x^{*}+u_{x} ; \\
& z=x^{*}+u .
\end{aligned}
$$

Here $x, y, z$ are observed; assume that $u$ is a Berkson type measurement error independent of $z$ with (unknown) density $f$, assume that $u_{y}, u_{x}$ have zero 
conditional (on $z$ and the other errors) expectations. For this model Newey (2001) proposed to consider an additional equation assuming that the conditional moment $E(g(x) x \mid z)$ exists. In the univariate case this gives a system of two equations with two unknown functions (as discussed in Schennach, 2007, Zinde-Walsh, 2009). Define $h(x)=x g(x)$, then

$$
\begin{aligned}
& g * f=w_{1}, \\
& h * f=w_{2},
\end{aligned}
$$

with $w_{1}=E(y \mid z), w_{2}=E(x y \mid z)$ known.

In the multivariate case for $x=\left(x_{1}, \ldots, x_{d}\right) \in R^{d}, w_{2 k}=E\left(x_{k} y \mid z\right), k=$ $1, \ldots, d, h_{k}(x)=x_{k} g(x)$ this can be generalized to equations (즈). If the density $f_{x^{*}}$ of $x^{*}$ were of interest, equation $f_{x^{*}}=f_{z} * f_{-u}$ could be added.

If the independence between the mismeasured variable and measurement error holds conditionally then the equation (2) can be written for densities conditional on some $x_{c}$, where if such densities exist this equation is

$$
\int g\left((z-u) \mid x_{c}\right) f\left(u \mid x_{c}\right) d u=w\left(z \mid x_{c}\right)
$$

Then equation (2) is defined for functions in spaces where the dimension or the argument is augmented by the dimension of the conditioning variable, $x_{c}$.

A common way of providing solutions to (2) and other equations is to 
consider them in some normed function spaces, e.g. of integrable functions such as $L_{p}$ or weighted $L_{p}$ spaces, e.g. Carrasco, Florens and Renault (2007). Solving the equations is often done by employing Fourier transforms. Since convolutions and Fourier transforms can be defined in different spaces, the question is which spaces are best suited for the problems.

Devroye and Győrfi (1985) view density from the perspective of $L_{1}$ space since the density (when it exists as a function) is absolutely integrable. However, in various problems of interest density may not exist, as in cases of measurement error for individuals answering survey questions (say, about income or consumption) where the probability of truthful reporting is nonzero and a mass point can arise $(\mathrm{Hu}, 2008)$. Density functions in $L_{1}$ do not necessarily converge even if the corresponding distribution functions converge uniformly. The way to overcome both the non-existence of density and convergence problems is to consider density as a generalized derivative of the distribution function as proposed in Zinde-Walsh (2008); this is done by defining the distribution function as a functional on a suitable space of well-behaved differentiable functions so that with this definition the distribution function inherits the good properties of the well-behaved functions and becomes differentiable (details in Zinde-Walsh, 2008, also see section 2.1 below); moreover, in the space of generalized functions the generalized densities converge if the distribution functions converge thus the problem of defining the density for a distribution is well-posed there.

Working in spaces of generalized functions also extends the classes of 
regression functions for which solutions can be obtained. In various applications the object of interest may be represented by "sum of peaks" function, such as a sum of delta functions see, e.g. Klann, Kuhn, Lorenz, Maass and Thiele (2007) where applications in astrophysics and mass spectroscopy are discussed; functions with sparse support or support that includes isolated points can arise in various applications. If $g$ represents a regression function, applying Fourier transform to the convolution equations in spaces of functions may require severe restrictions on the function. For example, in spaces of integrable functions (such as $L_{1}, L_{2}$ ) linear and polynomial regression functions as well as distribution functions in binary choice models would be excluded. Again, a natural extension is to consider spaces of generalized functions where Fourier transforms are defined for functions that can grow at polynomial rates as well as for objects with sparse support. Spaces of generalized functions were utilized by Klann et al (2007) for sum of peaks regression, by Zinde-Walsh (2008) for generalized density functions; by Schennach (2007) and Zinde-Walsh (2009) for the problem in errors in variables univariate regression model with possible polynomial growth in the function.

This paper examines convolution equations in generalized function spaces. The interest here focuses on the equation (2) where only the function $g$ is unknown (deconvolution) and the system of equations (3) with two unknown functions. The generalized functions spaces considered here are described in detail in Schwartz (1966) and Gel'fand and Shilov (1964).

Much of the paper is devoted to a theoretical development of the problem 
of solving these convolution equations in spaces of generalized functions: existence of a unique solution (identification) and continuity of the mapping from the known functions to the solution (well-posedness).

The usual blueprint for deconvolution in function spaces works as follows. Assume: $g * f=w$ holds, convolution exists, e.g. for functions in $L_{1}$, say, densities. Fourier transform $(F t)$ is defined: for the function $g, F t(g)=$ $\int g(x) \exp \left(i x^{T} \zeta\right) d x$; this is a characteristic function if $g$ is a density. Exchange formula applies: for Fourier transforms $\gamma=F t(g) ; \phi=F t(f) ; \varepsilon=F t(w)$ a convolution is transformed into product:

$$
g * f=\omega \Longrightarrow \gamma \phi=\varepsilon
$$

Also, if additionally Fourier transform exists for $h_{k}(x)=x_{k} g(x)$, then $F t\left(h_{k}\right)=$ $-i \frac{\partial}{\partial \xi_{k}} \gamma(\xi)$; denote the derivative $\frac{\partial}{\partial \xi_{k}} \gamma(\xi)$ by $\gamma_{k}^{\prime}$.

If $w$ and $f$ in equation (2) (thus also $\varepsilon$ and $\phi$ ) are known and $\phi \neq 0$ solve the algebraic equation : $\gamma=\phi^{-1} \varepsilon$, then apply the inverse Fourier transform, $F t^{-1}$, to obtain $g$

$$
g=F t^{-1}(\gamma)
$$

When the functions that enter the equations are estimated based on available data on the observables, the solutions will be stochastic and for establishing consistency well-posedness of the solutions becomes crucial; Carrasco et al (2007), An and $\mathrm{Hu}$ (2012) discuss well-posedness that applies to similar problems in various normed spaces, mostly in spaces of integrable functions, 
$L_{p}$

Thus for pursuing a similar approach in spaces of generalized functions the following questions are addressed here: 1. Under what assumptions do the convolution equations hold? 2. When is Fourier transform and its inverse defined? 3. When does the exchange formula hold (and when is a multiplicative product defined)? 4. When do transformed equations have a unique solution? 5. When is the problem well-posed, that is the solution continuously depends on the known generalized functions in the equation?

Once the conditions for identification and well-posedness are established in the generalized functions space, the question of consistent plug-in estimation can be examined. Suppose that the known functions, such as the densities (characteristic functions) of the observables, conditional expectations of the observables (and their Fourier transforms) in the models considered are consistently estimated; the solutions to the convolution equations based on these estimated functions are now random generalized functions; do they converge in some stochastic sense to the true function in the topology of generalized functions?

In order to answer this one needs to consider stochastic generalized functions which represent stochastic functionals on the spaces of well behaved (differentiable, etc.) functions. These are described in Gel'fand and Vilenkin (1964) and Koralov and Sinai (2007). The question of consistency requires providing conditions for stochastic convergence of Fourier transforms and inverse Fourier transforms, of derivatives and of products for random gen- 
eralized functions - all the operations that are involved in solving for the unknown functions.

Section 2 of this paper introduces the spaces of generalized functions considered here, gives conditions when convolutions are defined for generalized functions of interest, and when products of Fourier transforms are defined. Then the convolution theorem ("exchange formula") is provided making it possible to transform convolutions into products of Fourier transforms. Section 3 gives results on existence and uniqueness of solutions of the transformed equation or system of equations. These results give conditions for identification. General results on well-posedness of the solutions are proved here in Section 3 (some were previously given in the working paper Zinde-Walsh, 2009). Section 4 provides stochastic properties of gener-

alized functions. Section 5 gives conditions for consistent (in the topology of generalized functions) deconvolution and also for consistent non-parametric estimation of a regression function in the model (7-9).

\section{Convolution equations in generalized func- tions}

\subsection{Spaces of generalized functions}

Many different spaces of generalized functions can be defined; each may be best suited to some particular class of problems. This paper focuses on well 
known classical spaces of generalized functions discussed in the books by Schwartz (1966) and Gel'fand and Shilov (1964); reference is also made to related spaces as presented e.g. by Sobolev (1992).

Define a space of well behaved functions, sometimes called test functions; denote a generic such space by $G$. The functions in $G$ are defined on the real space, $R^{d}$, but may take values in the complex space since we consider characteristic functions and Fourier transforms that can take complex values. Two widely used spaces of test functions are $G=D$ and $G=S$.

The space $D$ is the linear topological space of infinitely differentiable functions each defined on a compact support, so that $D \subset C^{\infty}\left(R^{d}\right)$, where $C^{\infty}\left(R^{d}\right)$ is the space of all infinitely differentiable functions; to converge in $D$ the sequence of functions should be supported on a common bounded set and converge uniformly itself as well as have uniformly converging derivatives of all orders.

To define the space $S$ first introduce some notation. For any vector of non-negative integers $m=\left(m_{1}, \ldots m_{d}\right)$ and vector $t \in R^{d}$ denote by $t^{m}$ the

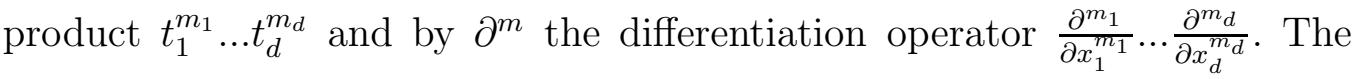
space $S \subset C^{\infty}\left(R^{d}\right)$ of rapidly decreasing functions is then defined as:

$$
S=\left\{\psi \in C^{\infty}\left(R^{d}\right):|t|^{m}\left|\partial^{l} \psi(t)\right|=o(1) \text { as } t \rightarrow \infty\right\},
$$

for any $d$-dimensional vectors of integers $m, l$, where $l=(0, \ldots 0)$ corresponds to the function itself, $|t|$ is the vector of absolute values of vector $t, t \rightarrow \infty$ 
coordinate-wise; thus the functions in $S$ go to zero at infinity faster than any power as do their derivatives of any order. A sequence in $S$ converges if in every bounded region each $|t|^{l}\left|\partial^{k} \psi(t)\right|$ converges uniformly.

The generalized functions space $G^{*}$ is the dual space, the space of linear continuous functionals on $G$. For any $b \in G^{*}$ and any $\psi \in G$ denote the value of the functional $b$ applied to $\psi$ by $(b, \psi)$. The topology is defined by weak convergence: a sequence $b_{n} \in G^{*}$ converges to $b \in G^{*}$ if for any $\psi \in G$ the sequence of the values of the functionals converges: $\left(b_{n}, \psi\right) \rightarrow(b, \psi)$.

Sobolev (1992) gives a general definition (in 1.8) where he points out a subtle distinction between the functional and a generalized function. Any generalized function, $b \in G^{*}$, can be defined by an equivalence class $\left\{b_{n}\right\}$ of weakly converging sequences of test functions $b_{n} \in G$ :

$b=\left\{\left\{b_{n}\right\}: b_{n} \in G\right.$, such that for any $\left.\psi \in G, \lim _{n \rightarrow \infty} \int b_{n}(t) \overline{\psi(t)} d t=(b, \psi)<\infty\right\}$,

where $\int \cdot d t$ denotes the multivariate integral over $R^{d}$, over-bar indicates complex conjugate for complex-valued functions and $(b, \psi)$ provides the value of the functional $b \in G^{*}$ for $\psi \in G$. However, the same functional can be represented by different generalized functions corresponding to different spaces $G$. For example, consider the $\delta$-function. This is a linear continuous functional on the space $C^{(0)}$ of continuous functions as well as on $D$ or $S$ and provides $(\delta, \psi)=\psi(0)$; it can be represented as an equivalence class of $\delta$-convergent sequences of continuous functions as well as of functions from $D$ or $S$. This 
implies that a generalized function considered as a functional can sometimes be extended to a linear continuous functional on a wider space.

Note that $D \subset S$ and thus there is the inclusion of the linear dual spaces: $S^{*} \subset D^{*}$; convergence in $S^{*}$ of linear continuous functionals implies their convergence in $D^{*}$, however, a sequence of elements of $S^{*}$ that converges in $D^{*}$ may not converge in the topology of $S^{*}$ (see the example in section 3.2).

In the terminology of Schwartz (1966) generalized functions are sometimes called "distributions" and elements of $S^{*}$ "tempered distributions"; here we shall call them generalized functions indicating the specific space considered. In Sobolev (1992, p.59) a diagram shows various chains of generalized functions spaces embedded in each other; these are spaces of functionals on spaces of continuously differentiable (of different orders) functions, continuously differentiable functions with compact support and Sobolev spaces.

Any locally summable (integrable on any bounded set) function $b(t)$ defines a generalized function $b$ in $D^{*}$ by

$$
(b, \psi)=\int b(t) \psi(t) d t
$$

on the space of real-valued test functions or by

$$
(b, \psi)=\int b(t) \overline{\psi(t)} d t
$$

for complex-valued functions. Any locally summable function $b(t)$ that ad- 
ditionally satisfies

$$
\int\left(\left(1+t^{2}\right)^{-1}\right)^{m}|b(t)| d t<\infty
$$

for some non-negative integer-valued vector $m=\left(m_{1}, \ldots, m_{d}\right)$ with $\left(\left(1+t^{2}\right)^{-1}\right)^{m}$ denoting the corresponding product $\prod_{i=1}^{d}\left(\left(1+t_{i}^{2}\right)^{-1}\right)^{m_{i}}$ similarly by (12) defines a generalized function $b$ in $S^{*}$; such generalized functions are called regular functions in the space of generalized functions.

Generalized derivatives $\partial^{m} b, m=\left(m_{1}, \ldots m_{d}\right)$ exist for any generalized function $b$ in $D^{*}$ and $S^{*}$ and are defined as functionals by the value for any function, $\psi$, as $\left(\partial^{m} b, \psi\right)=(-1)^{m}\left(b, \partial^{m} \psi\right)$ (here $\left.(-1)^{m}=(-1)^{m_{1}+\ldots+m_{d}}\right)$. The differentiation operator is continuous in $S^{*}$ and in $D^{*}$.

Thus, for example, a generalized density function, $f$, in the univariate case is defined as a generalized derivative of the regular distribution function, $F(x)$, by providing for any real-valued function, $\psi \in G$, the value

$$
(f, \psi)=-\int F(x) \frac{d \psi}{d x}(x) d x
$$

Thus defined generalized density is an element of $D^{*}$, or of $S^{*}$, moreover, it continuously depends on the distribution functions in the topology of either $S^{*}$ or $D^{*}$ by continuity of the differentiation operator. E.g. if $F(x)=I(x \geq$ 0 ) (the indicator function $I(\theta)=1$ if $\theta$ is true, zero otherwise), by substituting into (14) we obtain the generalized derivative as the Dirac $\delta$-function that provides $(\delta, \psi)=\psi(0)$.

Any generalized function can be represented as a generalized derivative 
of some order of a continuous function; to be an element of $S^{*}$ such a continuos function cannot grow faster than a polynomial. More specifically, by Schwartz (1966, theorem VI, p.239) any generalized function from $b \in S^{*}$ can be represented as a generalized derivative of a continuous function: $b=\partial^{l} c$ for a continuous function $c(x)$, which can be written as $c(x)=\left(1+x^{2}\right)^{\frac{m}{2}} \tilde{c}(x)$, where the continuous function $\tilde{c}(x)$ is uniformly bounded on $R^{d}:|\tilde{c}|<V$. Consider some fixed vectors of non-negative integers, $l$ and $m$ and a bound $V$. Denote by $S_{l, m}^{*}(V)$ the class of generalized functions, $b$, that have such a representation for $l, m$ and continuous $\tilde{c}$ : $|\tilde{c}(x)|<V$; any bounded in the topology of $S^{*}$ set of generalized functions has the representation $S_{l, m}^{*}(V)$ (see Schwartz, 1966, (VII,5;5), p.246-247). The smallest such $l$ is the order of integration that applied to a generalized function produces a continuous function and is related to the degree of singularity of the generalized function, while $m$ characterizes its growth at infinity.

The Fourier transform $(F t)$ is defined for functions in $D$, and more generally in $S$; it is an isomorphism of the space $S$; the value of the Fourier transform for the test function $\psi \in S$ also belongs to $S$ and its value at any $s \in R^{d}$ is $F t(\psi)(s)=\int \psi(x) e^{i x^{T} s} d x$ ( $x^{T}$ denotes transpose); in the dual spaces $D^{*}$ and $S^{*}$ the Fourier transform is given by $(F t(b), \psi)=(b, F t(\psi))$. The Fourier transform is an isomorphism of the space $S^{*}$.

For the analysis of this paper we mostly consider the functions of interest in the generalized functions space $S^{*}$, because of the fact that Fourier transform represents an isomorphism which permits to apply it or its inverse 
to any element, and the operator $F t$ and the inverse operator, $F t^{-1}$, are continuous in $S^{*}$.

Assumption 1. The generalized functions defined by the statistical model are in the space $S^{*}$.

This assumption allows for any distribution function on $R^{d}$ and does not require existence of density functions, for regression functions this allows growth at infinity, but limits it by (13), thus binary choice or polynomially growing regression functions are included, but not functions of exponential growth. Note that exponentially growing functions are included in the space $D^{*}$.

\subsection{Existence of convolutions; convolution pairs}

The convolution of generalized functions can be defined in different ways (see, e.g. Schwartz, 1966, p.154 or Sobolev, 1992, p. 63; Gel'fand and Shilov, 1964, v. I, p.103-104); it does not always have meaning and exists for specific pairs of mutual convolutors.

Consider the following spaces of test functions and of generalized functions on $R^{d}: \quad D, S, C^{\infty}, \mathcal{O}_{M}, D^{*}, S^{*}, E^{*}, \mathcal{O}_{C}^{*}$, where $C^{\infty}=C^{\infty}\left(R^{d}\right)$ is the space of infinitely differentiable functions on $R^{d} ; \mathcal{O}_{M} \subset C^{\infty}$ is the subspace of infinitely differentiable functions with every derivative growing no faster than a polynomial, $E^{*}$ is the subspace of generalized functions with compact support, and $\mathcal{O}_{C}^{*}$ is the subspace of rapidly decreasing (faster than any

polynomial) generalized functions (Schwartz, 1966, p.244). Table 1 shows 


$\begin{array}{ccccccc}g \backslash f & D & S & E^{*} & O_{C}^{*} & S^{*} & D^{*} \\ D & D & S & D & S & O_{M} & C_{\infty} \\ S & S & S & S & S & O_{M} & X \\ E^{*} & D & S & E^{*} & O_{C}^{*} & S^{*} & D^{*} \\ O_{C}^{*} & S & S & O_{C}^{*} & O_{C}^{*} & S^{*} & X \\ S^{*} & O_{M} & O_{M} & S^{*} & S^{*} & X & X \\ D^{*} & C_{\infty} & X & D^{*} & X & X & X\end{array}$

pairs of spaces for elements of which convolution is defined ( $\mathrm{X}$ indicates that convolution cannot be defined for some pairs of elements of the spaces); the table entries indicate to which space the element resulting from the convolution operation belongs. The table is an extended version of the one in the textbook by Kirillov and Gvishiani (1982, p.102) and summarizes the well-established results in the literature.

The convolution pairs in the table where convolution is defined all possess the hypocontinuity property (Schwartz, 1966, p.167, p.247-257). Hypocontinuity of a bilinear operation means that if one component of a pair is in a bounded set in $G^{*}$ and the other converges to zero in $G^{*}$, the result of the bilinear operation converges to zero (Schwartz, 1966, pp.72,73).

Convolution of a pair of arbitrary generalized functions is not always defined in $S^{*}$. Bounded support or at least rapid decline at infinity is needed for a convolution to exist. For example, convolution of a constant function with another constant function on $R^{1}$ is not defined. Nevertheless there are pairs of subspaces of generalized functions beyond those in the Table for which convolution defines a generalized function; spaces where convolution is 
defined can be combined. The convolution is a bilinear operation (Schwartz, 1966, p.157); convolution of a tensor product of generalized functions on two vector spaces, $R^{d_{1}}, R^{d_{2}}$ is the tensor product of the convolutions of functions in each space (Schwartz, 1966, p.158). Moreover convolution of any number of generalized functions can be defined in $D^{*}$ as long as all except possibly one have compact supports and this operation is associative and commutative (Schwartz, 1966, p.158); a variable shift or derivative of a convolution exists and is obtained by a shift or differentiation of any of the generalized functions entering the convolution (Schwartz, 1966, p.160).

Definition Call a pair of subspaces of generalized functions, $A \subset G^{*}$ and $B \subset G^{*}$ a convolution pair $(A, B)$ if for any $a \in A, b \in B$ convolution $a * b$ is defined in $G^{*}$; it is a hypocontinuous operation in the topology of $G^{*}$.

Note that if $(A, B)$ is a convolution pair then $(A \cup G, B \cup G)$ is also a convolution pair.

All the pairs of spaces in the Table satisfy this definition.

Assumption 2. The statistical model defines functions, $g$ and $f$ in $G^{*}$ such that $g \in A \subset G^{*}$ and $f \in B \subset G^{*}$; the subspaces $(A, B)$ form a convolution pair.

This assumption implies that (2) holds; it is often satisfied in statistical problems. Convolution of generalized density functions exists, thus (11) leads to (2) for all distributions even when the density functions do not exist in the ordinary sense. The finite sum of $\delta$-functions considered by Klann et al $(2007)$ is in $E^{*}$, thus convolution with any element of $D^{*}$ (or $S^{*}$ ) exists 
in $D^{*}\left(S^{*}\right)$. Schennach $(2007)$ considered univariate errors in variables model with instrumental variables and a regression function, $g$, bounded by polynomials; these regression functions are in $S^{*}$, convolution with any generalized density function from $\mathcal{O}_{C}^{*}$ exists in $S^{*}$. For regression functions $g$ in subspaces of $S^{*}$ where growth is more restricted, convolution with less rapidly declining $f$ may exist.

For a generalized function $g$ denote by $h_{k}$ the product of $g$ by the $k$ th component of $x \in R^{d}, x_{k}$, for a regular function $g(x)$ this is $h_{k}(x)=x_{k} g(x)$. In many cases when the convolution $g * f$ is defined, the convolution $h_{k} * f$ is also defined. Indeed, this is so in all the examples above: if $g$ has compact support, so does any $h_{k}$; if $g \in C^{\infty}$, it is true of $h_{k}$ as well, etc. Thus some models accommodate not only (2), but also other equations, e.g. providing (3).

Assumption 3. The statistical model is such that in addition to Assumption $2, h_{k} \in A, k=1, \ldots, d$.

\subsection{Fourier transforms, exchange formula and some special convolution pairs}

Next, consider the Fourier transforms and the exchange formula.

For the generalized functions in equations (3) denote Fourier transforms as $\gamma=F t(g), \phi=F t(f)$ and $\varepsilon_{i}=F t\left(w_{i}\right)$. Recall that the Fourier transform always exists in $S^{*}$ and is a continuous isomorphism. 
The classical case of the convolution pair $\left(S^{*}, \mathcal{O}_{C}^{*}\right)$ is examined in Schwartz (1966). If $(g, f)$ belong to $\left(S^{*}, \mathcal{O}_{C}^{*}\right)$ the convolution exists and equation (2) transforms into the multiplicative equation $\gamma \phi=\varepsilon$, where $\gamma \in S^{*}, \phi \in \mathcal{O}_{M}$ and $\varepsilon \in S^{*}$ (Schwartz, 1966, p.281-282). The product between a generalized function in $S^{*}$ and a function from $\mathcal{O}_{M}$ always exists in $S^{*}$; multiplication is a hypocontinuous operation (Schwartz, 1966, p.243-246). Thus there is a dichotomous relation between the classical convolution pair $\left(S^{*}, \mathcal{O}_{C}^{*}\right)$ and the product pair of generalized functions spaces, $\left(S^{*}, \mathcal{O}_{M}\right)$. Below we show that this dichotomy extends to other convolution pairs of spaces.

Define a product pair of spaces as a pair $(\Gamma, \Phi)$ of subspaces of $S^{*}$ such that for any $\gamma \in \Gamma, \phi \in \Phi$ the product $\gamma \phi$ defines an element $\varepsilon$ in $S^{*}$; the operation of multiplication for $(\Gamma, \Phi)$ is hypocontinuous in the topology of $S^{*}$.

Theorem 1 If Assumptions 1 and 2 are satisfied then for any $(g, f) \in(A, B)$ the exchange formula applies.

Proof. Consider the special sequences studied by Mikusinski (Antosik et al, 1973) and Hirata and Ogata (1958) that are defined for a generalized function $b$ as $\tilde{b}_{n}=b * \delta_{n}$ with $\delta_{n}$ representing the following delta-convergent sequence: for a number sequence: $\alpha_{n}>0$ and $\alpha_{n} \rightarrow 0$, the regular function $\delta_{n}(x)$ is non-negative with support in $|x|<\alpha_{n}$ and $\int \delta_{n}(x) d x=1$; the convolution with $\delta_{n} \in E^{*}$ is always defined. Moreover, $\tilde{b}_{n} \rightarrow b$ in $S^{*}$ : indeed, $\left(b * \delta_{n}, \psi\right)=\left(b, \psi * \delta_{n}\right)$, but $\psi * \delta_{n}$ is in $S$ and converges there to $\psi$, so 
$\left(b * \delta_{n}, \psi\right)$ converges to $(b, \psi)$.

Since $g, f$ belong to a convolution pair of spaces $(A, B)$, we can enlarge the convolution pair $(A, B)$ to include with every $b \in A$ or $B$ the corresponding sequences $\tilde{b}_{n}$. Denote the resulting pair of spaces by $(\tilde{A}, \tilde{B})$. Show that this is also a convolution pair. Note that the convolution $\tilde{g}_{n} * \tilde{f}_{n}$ is defined as support of $\delta_{n}$ is bounded. All that is needed is to show hypocontinuity; it follows from the fact that if a set $T$ from $A$ or $B$ is bounded in $S^{*}$ so is the corresponding set $\tilde{T}$ that contains every element $b \in T$ as well as all $\tilde{b}_{n}$. If a sequence $b_{m}$ converges to zero in $S^{*}$ so does the corresponding $\tilde{b}_{m n}$, and hypocontinuity extends to the enlarged convolution pair.

To show the exchange formula we need to establish that for any $(g, f) \in$ $(A, B)$ we get that $F t(g) F t(f)=F t(g * f)$.

Start by the exchange formula of Hirata and Ogata for the sequences:

$$
\lim F t\left(\tilde{g}_{n} * \tilde{f}_{n}\right)=\lim F t\left(\tilde{g}_{n}\right) \lim F t\left(\tilde{f}_{n}\right) .
$$

Consider first the left-hand side; by the continuity of Fourier transform in $S^{*}$

$$
\lim F t\left(\tilde{g}_{n} * \tilde{f}_{n}\right)=F t\left(\lim \left(\tilde{g}_{n} * \tilde{f}_{n}\right)\right),
$$

then by hypocontinuity of the convolution and because $\tilde{f}_{n} \rightarrow f, \tilde{g}_{n} \rightarrow g$ we get that this is $F t(g * f)$. 
On the right hand side by continuity of Fourier transform and convergence

$$
\lim F t\left(\tilde{g}_{n}\right) \lim F t\left(\tilde{f}_{n}\right)=F t(g) F t(f) .
$$

Denote the space of Fourier transforms of elements from $A$ (or $B$ ) by Ft $(A$ ) (correspondingly, $F t(B))$. Then $(F t(A), F t(B))$ is a product pair. Hypocontinuity of the product follows immediately from the hypocontinuity of the convolution and continuity of the Fourier transform and its inverse.

Corollary 1. Given a product pair of spaces $(\Gamma, \Phi)$ in $S^{*}$ the exchange formula applies to any $\gamma, \phi \in(\Gamma, \Phi)$ :

$$
F t^{-1}(\gamma \phi)=F t^{-1}(\gamma) * F t^{-1}(\phi) .
$$

The proof follows the proof of Theorem 1 above by replacing convolution with product and Fourier transform by the inverse Fourier transform.

In examining problems such as deconvolution much of the literature focuses on Fourier transforms, e.g. characteristic functions. The dichotomy between convolution pairs of spaces and product pairs of spaces in $S^{*}$ allows to switch between the two types of pairs.

If $\phi$ is the characteristic function of a measurement or contamination error the condition $\phi \in \mathcal{O}_{M}$ for the classical product pair it would require existence of all moments. It may be of interest to consider pairs where products of Fourier transforms of generalized functions exist for less smooth functions (e.g. with relaxed moments requirements on measurement error); 
relaxing the smoothness of $\phi$ will require restricting the degree of singularity of $\gamma$.

For a continuous function $\phi \in C^{(0)}$ define the space of test functions, denoted $G \oplus \phi G$, that consists of functions that can be represented as $\psi_{1}+\phi \psi_{2}$, with $\psi_{i} \in G(G=D$ or $S)$. Consider $\gamma \in G^{*}$ that can be extended to a continuous linear functional on $G \oplus \phi G$, denote the linear space generated by such $\gamma$ by $G(\phi)^{*}$. If $\phi \in G$ then $G(\phi)^{*}=G^{*}$. For any $\psi \in G$ the value $(\gamma, \phi \psi)$ is defined and is a continuous functional with respect to $\psi$; this defines the generalized function $\phi \gamma:(\phi \gamma, \psi)=(\gamma, \phi \psi)$. Then $\left(G(\phi)^{*}, G \oplus \phi G\right)$ is a product pair; the corresponding spaces of inverse Fourier transforms form a convolution pair.

For example, the derivative, $\delta^{\prime}$, of a univariate Dirac $\delta$-function in $G^{*}$ can be multiplied by any continuous function, $\phi$, that is differentiable at 0 , since then $\left(\delta^{\prime} \phi, \psi\right)=\left(\delta^{\prime}, \phi \psi\right)$ with $\left(\delta^{\prime}, \phi \psi\right)=\phi^{\prime}(0) \psi(0)+\phi(0) \psi^{\prime}(0)$. More generally, for a product between a continuous function and a generalized function to be defined, there is a trade-off between differentiability of the continuous function and the degree of singularity of the generalized function.

Under the Assumptions 1-3 the convolution equations (2:31) lead to corresponding equations for Fourier transforms:

$$
\gamma \cdot \phi=\varepsilon
$$


or to the system of equations

$$
\begin{aligned}
\gamma \cdot \phi & =\varepsilon_{1} \\
\gamma_{k}^{\prime} \cdot \phi & =i \varepsilon_{2 k}, k=1, \ldots, d .
\end{aligned}
$$

\section{Solutions to the convolution equations: iden- tification and well-posedness}

\subsection{Identification}

For identification the supports of the functions in the equations play an important role.

Recall that for a continuous function $\psi(x)$ on $R^{d}$ support is defined as the set $W=\operatorname{supp}(\psi)$, such that

$$
\psi(x)=\left\{\begin{array}{cc}
a \neq 0 & \text { for } x \in W \\
0 & \text { for } x \in R^{d} \backslash W
\end{array}\right.
$$

Support of a continuous function is an open set.

Since generalized functions can be considered as functionals on the space $S$ support of a generalized function $b \in S^{*}$ is defined as follows (Schwartz, 1966, p. 28). Denote by $(b, \psi)$ the value of the functional $b$ for $\psi \in S$. Consider open sets $W$ with the property that for any $\psi \in S: \operatorname{supp}(\psi)=W$ the value of the functional $(b, \psi)=0$; then define the null set for $b$ as the union 
of all such sets: $\Omega=\cup W$. Then $\operatorname{supp}(b)=R^{d} \backslash \Omega$. Note that a generalized function has support in a closed set, for example, support of the $\delta$-function is just one point 0 .

We start with deconvolution for (2). In the deconvolution problem $f$, or equivalently its Fourier transform, $\phi$, is assumed to be given. Typically, $\phi$ is a characteristic function and thus is continuous and bounded.

The convolution equation (2) uniquely identifies $g$ for a known $f$ if it can be shown that the corresponding equation (15) has meaning and can be uniquely solved for $\gamma$.

Next a useful Lemma is proved. This Lemma shows that if a product between a generalized function $\gamma$ and a continuous function $\phi$ is defined: $\varepsilon=\gamma \phi$, then division of $\varepsilon$ by $\phi$ is uniquely defined on $\operatorname{supp}(\phi)$ in $D^{*}$.

Lemma 1. Suppose that $\varepsilon=\gamma \phi$ in $D^{*} ; \phi$ is a continuous function. Then the generalized function $\phi^{-1} \varepsilon$ is uniquely defined in $D^{*}$ on $\operatorname{supp}(\phi)$.

Proof. Denote $W=\operatorname{supp}(\phi)$ and consider $D(W)$ the space of all the functions in $D$ with supports restricted to belong to $W$; the space $D^{*}(W)$ is the dual space for $D(W)$. Next, consider a covering of the open set $W$ by bounded sets: $W=\cup W_{\nu}$ where each $W_{\nu}$ is an open bounded set. Similarly, consider $D\left(W_{\nu}\right)$. Then any generalized function in $D^{*}$ can be restricted to the dual space $D^{*}\left(W_{\nu}\right)$.

In $D^{*}\left(W_{\nu}\right)$ the generalized function $\gamma$ solves $\varepsilon=\gamma \phi$. Suppose that the solution is not unique and there is $\tilde{\gamma} \neq \gamma$ such that $\tilde{\gamma} \phi=\varepsilon$. Then $(\gamma-\tilde{\gamma}) \phi$ is defined and represents a zero element in $D^{*}\left(W_{\nu}\right)$. A zero functional can 
be extended from the space $D\left(W_{\nu}\right)$ to a zero functional on the space of continuous functions on $W_{\nu}, C^{(0)}\left(W_{\nu}\right) \subset C^{(0)}\left(R^{d}\right)$. Then $((\gamma-\tilde{\gamma}) \phi, \psi)=0$ for any $\psi \in C^{(0)}\left(W_{\nu}\right)$, but since $\phi$ is invertible as a continuous function on $W_{v}$ any continuous function $\psi$ in $C^{(0)}\left(W_{\nu}\right)$ has the representation $\phi \tilde{\psi}$ for some $\tilde{\psi} \in C^{(0)}\left(W_{\nu}\right)$. This implies that $(\gamma-\tilde{\gamma}, \psi)$ is defined (equals zero) for any $\psi$ thus the functional $\gamma-\tilde{\gamma}$ extends to $C^{(0)}\left(W_{\nu}\right)$ as a zero functional. This is only possible if $\gamma-\tilde{\gamma}$ is a zero functional in $D^{*}\left(W_{\nu}\right)$. Then $\gamma-\tilde{\gamma}$ is a zero generalized function in $D^{*}(W)$.

Recall that the difference between $D^{*}$ and $S^{*}$ is in the "tail behavior" only; on bounded sets the two spaces coincide. The following theorem provides deconvolution in $S^{*}$.

Theorem 2 Under Assumptions 1 and 2 assume that $\phi=F t(f)$ is a known continuous function; then for any $W$ where supp $(\phi) \supset W$, the Fourier transform of $g, \gamma$, is uniquely defined on $W$; if it is further known that $\operatorname{supp}(\gamma)=$ $W$, then $g$ is uniquely defined in $S^{*}$. Uniqueness holds automatically if $\operatorname{supp}(\phi)=R^{d}$

Proof. By Theorem 1, (15) holds in $S^{*}$. Since $\operatorname{supp}(\phi) \supset W$ by Lemma 1 a unique solution $\gamma$ to (15) exists in $D^{*}(W)$. Since $\gamma$ is the Fourier transform of $g \in S^{*}, \gamma \in S^{*}$ and defines an element in $S^{*}(W) \subset D^{*}(W)$ uniquely. If support of $\gamma$ is restricted to $W$ a priori, then the solution is unique, thus when $\operatorname{supp}(\phi)=R^{d}$, the generalized function $\gamma$ is defined uniquely in $S^{*}$. An inverse Fourier transform exists in $S^{*}$ for any $\gamma$. It is then possible to recover 
$g$ by the inverse Fourier transform $g=F t^{-1}(\gamma)$, thus $g$ is uniquely defined whenever $\gamma$ is.

Generally by this Theorem identification in deconvolution holds whenever $\operatorname{supp}(\phi)=R^{d}$. However, this excludes some error distributions such as the uniform or the triangular, where the characteristic function has isolated zeros. A more general result (Schwartz, 1966, pp.123-125) establishes the possibility of division by $\phi$ even when $\phi$ has zeros. In the one-dimensional case as long as $\phi$ is infinitely differentiable, the zeros are isolated and there exists a finite order derivative that is non-zero at every zero point of $\phi$, any generalized function can be divided by $\phi$. Thus since the uniform and triangular distributions have this property, deconvolution with these error distributions is also identified in $S^{*}$.

The next Theorem examines identification in the case when there are two unknown functions in the system (3) ; under Assumptions 1-3 it leads to (16). The main conditions are continuous differentiability of one of $\gamma$, or $\phi$, assumptions about support and knowledge of the value of the differentiable function at an interior point. If that function is a characteristic function, its value at 0 is always 1 .

Theorem 3 Under Assumptions 1-3 for the system of equations (16) if $\operatorname{supp}(\phi) \supset \operatorname{supp}(\gamma)=W$, where $W$ is a connected set in $R^{d}$ that includes 0 as an interior point, and

(a) if $\gamma$ is continuously differentiable in $W, \gamma(0)=c$, then $\gamma$ is uniquely 
defined on $W$ by

$$
\gamma(s)=c \exp \int_{0}^{s} \sum_{k=1}^{d} \varkappa_{k}(t) d t_{k}
$$

with the uniquely defined continuous functions $\varkappa_{k}$ that solve

$$
\varkappa_{k} \varepsilon_{1}-i \varepsilon_{2 k}=0, k=1, \ldots, d
$$

or

(b) if $\phi$ is continuously differentiable in $W, \phi(0)=1$, then $\gamma$ is uniquely defined on $W$ by

$$
\gamma=\tilde{\phi}^{-1} \varepsilon_{1}
$$

where

$$
\tilde{\phi}(s)=\exp \int_{0}^{s} \sum_{k=1}^{d} \tilde{\varkappa}_{k}(t) d t_{k}
$$

with the uniquely defined on $W$ continuous functions $\tilde{\varkappa}_{k}$ that solve

$$
\varepsilon_{1} \tilde{\varkappa}_{k}-\left(\left(\varepsilon_{1}\right)_{k}^{\prime}-i \varepsilon_{2 k}\right)=0, k=1, \ldots, d \text {. }
$$

Then $g=F t^{-1}(\gamma)$. If $\operatorname{supp}(\gamma)=W$, then $g$ is uniquely defined. Uniqueness holds automatically if $W=R^{d}$. If $\operatorname{supp}(\phi)=W$, then $\phi$ is also uniquely defined; and so is $f=F t^{-1}(\phi)$.

Proof. (a) Consider the space of generalized functions $D^{*}(W)$ (defined in proof of Lemma 1). Since continuous $\gamma$ is non-zero on $W$ by Lemma 1 (reversing the roles of $\phi$ and $\gamma$ there) in $D^{*}(W)$ the generalized function 
$\phi$ is uniquely expressed as $\gamma^{-1} \varepsilon_{1}$. Substitute this expression for $\phi$ into the differential equations in (16), and denote the continuous functions $\gamma_{k}^{\prime} \gamma^{-1}$ by $\varkappa_{k}(\xi)$ to obtain equations

$$
\varkappa_{k}(\xi) \varepsilon_{1}-i \varepsilon_{2 k}=0, k=1, \ldots, d
$$

where the left-hand side is defined and equals zero in $S^{*}$.

We can show that the function $\varkappa_{k}$ is uniquely determined in the class of continuous functions on $W$ by the equation (19). Proof is by contradiction. Suppose that there are two distinct continuous functions on $\operatorname{supp}(\gamma), \varkappa_{k 1} \neq$ $\varkappa_{k 2}$ that satisfy $((19))$. Then $\varkappa_{k 1}(\bar{x}) \neq \varkappa_{k 2}(\bar{x})$ for some $\bar{x} \in \operatorname{supp}(\gamma)$. Without loss of generality assume that $\bar{x}$ is in the interior of $W$; by continuity $\varkappa_{k 1} \neq$ $\varkappa_{k 2}$ everywhere for some closed convex $U \subset W$. Consider now $D(U)^{*}$; we can write

$$
\left(\varepsilon_{1}\left(\varkappa_{k 1}-\varkappa_{k 2}\right), \psi\right)=0
$$

for any $\psi \in D(U)$. A generalized function that is zero for all $\psi \in D(U)$ coincides with the ordinary zero function on $U$ and is also zero for all $\psi \in$ $D_{0}(U)$, where $D_{0}(U)$ denotes the space of continuous test functions on $U$. For the space of test functions $D_{0}(U)$ multiplication by continuous $\left(\varkappa_{k 1}-\varkappa_{k 2}\right) \neq 0$ is an isomorphism. Then we can write

$$
0=\left(\left[\varepsilon_{1}\left(\varkappa_{k 1}-\varkappa_{k 2}\right)\right], \psi\right)=\left(\varepsilon_{1},\left(\varkappa_{k 1}-\varkappa_{k 2}\right) \psi\right)
$$


implying that $\varepsilon_{1}$ is defined and is a zero generalized function in $D_{0}(U)^{\prime}$. If that were so $\varepsilon_{1}$ would be a zero generalized function in $D(U)^{*}$ since $D(U) \subset D_{0}(U)$ but this is not possible since $\varepsilon_{1}=\gamma \phi$, which is non-zero by assumption.

Next we show that $\gamma$ is then uniquely determined on $W$. Indeed, for any $t, s \in \operatorname{supp}(\gamma)$ with the $k t h$ coordinate denoted $t_{k}$, write the continuous function

$$
\gamma(s)=c \exp \int_{0}^{s} \sum_{k=1}^{d} \varkappa_{k}(t) d t_{k}
$$

where integration is along any arc joining 0 and $s$ in $W$. This is the unique solution to $\gamma(0)=c, \gamma^{-1} \gamma_{k}^{\prime}=\varkappa_{k}$ (see, e.g., Schwartz, 1966, p.61). Then in $S^{*} g=F t^{-1}(\gamma)$ is uniquely defined.

(b) In view of the result in Theorem 2 it is sufficient to show that $\phi$ is uniquely determined on $W$. Consider the space of generalized functions $D^{*}(W)$. Since $\phi$ is non-zero on $W$ and continuously differentiable, then by differentiating the first equation in (16), substituting from the second equation and multiplying by $\phi^{-1}$ in $D^{*}(W)$ (where the product exists as shown in Lemma 1) we get that the generalized function

$$
\varepsilon_{1} \phi^{-1} \phi_{k}^{\prime}-\left(\left(\varepsilon_{1}\right)_{k}^{\prime}-i \varepsilon_{2 k}\right)
$$

equals zero in the sense of generalized functions, in $D^{*}(W)$. Note that by assumption $\varepsilon_{1}$ cannot be zero on $W$ and both $\varepsilon_{1}$ and $\varepsilon_{2 k}$ are zero outside of $W$. Define $\tilde{\varkappa}_{k}=\phi_{k}^{\prime} \phi^{-1}$; then $\tilde{\varkappa}_{k}$ is continuous on $\operatorname{supp}(\gamma)$ and is a regular 
function that satisfies the equation

$$
\varepsilon_{1} \tilde{\varkappa}_{k}-\left(\left(\varepsilon_{1}\right)_{k}^{\prime}-i \varepsilon_{2 k}\right)=0
$$

We can show that the function $\tilde{\varkappa}_{k}$ is uniquely determined in the class of continuous functions on $W$ by the equation (20). Proof is identical to the proof in part (a) for equation $((19))$. Next we show that $\phi$ is then uniquely determined on $W$. Indeed, the continuous function

$$
\tilde{\phi}(\zeta)=\exp \int_{0}^{s} \sum_{k=1}^{d} \varkappa_{k}(t) d t_{k}
$$

is the unique solution to $\tilde{\phi}(0)=1, \tilde{\phi}_{k}^{-1} \tilde{\phi}^{\prime}=\varkappa_{k}$; then since $\varkappa_{k}\left(=\phi_{k}^{\prime} \phi^{-1}\right)$ is uniquely determined on $W$, so is $\phi$ on $W$ where it coincides with $\tilde{\phi}$.

By Theorem 2, then $\gamma$ is then uniquely defined on $W$. Then in $S^{*} g=$ $F t^{-1}(\gamma)$ is uniquely defined.

This Theorem extends the identification results of Schennach (2007) and Zinde-Walsh(2009) to the multivariate case and to the case when $\gamma$ rather than $\phi$ is continuously differentiable (part (a) of this Theorem), and extends the identification result of Cunha et al (2011) by showing that in the model with several measurements considered there identification additionally holds with the requirement of continuous differentiability of $\phi$ replacing the requirement that $\gamma$ be continuously differentiable (part (b)). 


\subsection{Well-posedness of the deconvolution in $S^{*}$}

Well-posedness requires that a unique solution to the problem exist and that this solution be continuous in some "reasonable topology" (Hadamard, 1923). Here most of the results consider the topology of generalized functions which is weaker than, say, the uniform or $L_{1}$ norm for corresponding subspaces, thus well-posedness may hold in this topology when it does not hold in the usual norms. But well-posedness may not always obtain even in this weak topology. An example is provided that involves deconvolution with a supersmooth distribution.

Consider a convolution pair of spaces $(A, B)$ in $S^{*}$, denote by $C$ the space of elements in $S^{*}$ represented by convolutions of generalized functions from $(A, B)$. Well-posedness of the deconvolution would require that for the given $f \in B$ and any sequence of $w_{n} \in C$ that converges to some $w \in C$ in the topology of $S^{*}: w_{n} \rightarrow w$, the sequence of $g_{n} \in A$ such that $g_{n} * f=w_{n}$ would converge to $g \in A$, with $g * f=w$. By Theorem 1 this convergence can be restated for the product pair of spaces $(\Gamma, \Phi)$ where the product pair is the image of the convolution pair $(A, B)$ under Fourier transform; denote by $\Pi \in S^{*}$ the space of products of elements from the pair $(\Gamma, \Phi)$. Then wellposedness can be restated in terms of the Fourier transforms: the problem is well-posed in $S^{*}$ if for any $\varepsilon_{n} \rightarrow \varepsilon$ where $\varepsilon_{n}, \varepsilon \in \Pi$ the corresponding sequence $\gamma_{n}=\phi^{-1} \varepsilon_{n}$ converges to $\gamma=\phi^{-1} \varepsilon$.

If $\phi^{-1} \in O_{M}$ then by hypocontinuity of the product, for any sequence $\varepsilon_{n}-\varepsilon$ that converges to zero in $S^{*}$ the corresponding sequence $\gamma_{n}-\gamma$ also 
converges to zero and well-posedness obtains without any restrictions on $\gamma$. This result could also extend to infinitely differentiable $\phi$ with some zeros and applies in the univariate deconvolution to error distributions such as the uniform and triangular (see Schwartz, pp. 123-125).

Below we provide cases of well-posed deconvolution where less restrictive differentiability conditions are imposed on $\phi$; this would require additional conditions on the $\gamma^{\prime}$ s. The nature of the conditions is to ensure that the product pair is defined for the Fourier transforms; for a continuous $\phi$ this requires a trade-off between the degree of singularity of $\gamma$ (and correspondingly, $\varepsilon$ ) and the differentiability of $\phi$, these trade-offs can occur locally as in the example where $\gamma$ is the derivative of a $\delta$-function and $\phi$ is continuously differentiable at 0 , but to streamline the proofs we consider global restrictions in the product pairs.

First, suppose that both $\phi$ and $\gamma$ are continuos functions, then $\varepsilon$ is continuous as well. Let $\Gamma$ be a subspace of all continuous functions on $R^{d}$ such that all $\gamma \in \Gamma$ belong in a bounded set in $S^{*}$; then for some $\bar{m}$ we have $\Gamma=S_{0, \bar{m}}^{*}(V)$ (implying $\left.\left(\left(1+t^{2}\right)^{-1}\right)^{\bar{m}}|\gamma|<V<\infty\right)$. Then for any bounded continuous $\phi$ the product $\varepsilon=\gamma \phi \in S_{0, \bar{m}}^{*}(V)$.

Lemma 2. Suppose that $\gamma, \gamma_{n} \in S_{0, \bar{m}}^{*}(V), \phi$ is a bounded continuous function; $\varepsilon_{n}=\gamma_{n} \phi, \varepsilon=\varepsilon_{0}=\gamma \phi, 0 \leq n<\infty$ and $\phi^{-1}$ is a continuous function that satisfies (13). Then if $\varepsilon_{n} \rightarrow \varepsilon_{0}$ as $n \rightarrow \infty$ in $S^{*}$ we get that $\gamma_{n}=\phi^{-1} \varepsilon_{n}$ converges to $\gamma=\phi^{-1} \varepsilon_{0}$.

Proof. First note that for any number $\xi>0$ for any $\psi \in S$ there exists 
some bounded set $\Lambda \in R^{d}$ such that

$$
\left|\int_{R^{d} \backslash \Lambda} \varepsilon_{n}(t) \phi^{-1}(t) \psi(t) d t\right|<\xi .
$$

Indeed, $\left|\int_{R^{d} \backslash \Lambda} \varepsilon_{n}(t) \phi^{-1}(t) \psi(t) d t\right| \leq$

$$
\begin{aligned}
& V \int_{R^{d} \backslash \Lambda}\left(\left(1+t^{2}\right)^{-1}\right)^{-(\bar{m}+m)}\left|\phi^{-1}(t)\right|\left|\left(\left(1+t^{2}\right)^{1}\right)^{m} \psi(t)\right| d t \\
\leq & V \sup _{R^{d} \backslash \Lambda}\left|\left(\left(1+t^{2}\right)^{1}\right)^{m} \psi(t)\right| \int_{R^{d}}\left(\left(1+t^{2}\right)^{-1}\right)^{-m}\left|\phi^{-1}(t)\right| d t .
\end{aligned}
$$

Since by (13) $\int_{R^{d}}\left(\left(1+t^{2}\right)^{-1}\right)^{-m}\left|\phi^{-1}(t)\right| d t$ is bounded, say, by some $V_{\phi}$ and for $\psi \in S$ as $t \rightarrow \infty$ the value $\left|\left(\left(1+t^{2}\right)^{1}\right)^{m} \psi(t)\right|$ converges to zero, the set $\Lambda$ can be selected such that

$$
\sup _{R^{d} \backslash \Lambda}\left|\left(\left(1+t^{2}\right)^{-1}\right)^{m} \psi(t)\right|<\xi V_{\phi}^{-1} V^{-1}
$$

Consider now the value of the functional $\left(\varepsilon_{n} \phi^{-1}-\varepsilon \phi^{-1}, \psi\right)$. Since the sequence of continuous bounded functions $\varepsilon_{n}-\varepsilon$ converges to zero in $S^{*}$ it converges to zero point-wise and uniformly on bounded $\Lambda$. Then for any $\zeta$ and $\Lambda$ corresponding to $\xi=\frac{1}{2} \zeta$ we can find $N$ such that

$$
\sup _{\Lambda}\left|\varepsilon_{n}-\varepsilon_{0}\right|\left|\int \phi^{-1}(t) \psi(t) d t\right|<\xi
$$

for any $n>N$. It follows that $\left|\left(\phi^{-1} \varepsilon_{n}-\phi^{-1} \varepsilon, \psi\right)\right|<\zeta$.

Thus $\left|\gamma_{n}-\gamma\right| \rightarrow 0$ in $S^{*}$. 
In the measurement error problem the function $\varepsilon$ is a characteristic function; if all $\varepsilon_{n}$ in the equation (15) are characteristic functions or at least some continuous and uniformly bounded functions then they satisfy the conditions of Lemma 2 with $\bar{m}=0$.

The somewhat unusual requirement that the sequence that converges be bounded in $S^{*}$ is a requirement that is associated with the weak topology of the space of generalized functions. In normed spaces convergence implies eventual boundedness in norm of the converging sequence, but this is not the case for generalized functions that do not exclude singularities and polynomial growth. The boundedness requirement uniformly limits the degree of singularity and the rate of divergence at infinity of the generalized functions in the bounded set. It is however less restrictive than many assumptions in normed spaces such as boundedness or integrability.

The Lemma demonstrates that unlike the behavior in function spaces where the deconvolution is usually ill-posed, in the weaker topology of the space $S^{*}$ deconvolution is well-posed when the error distribution is such that the condition (13) is satisfied. Usually well-posedness in deconvolution is examined in terms of ordinary smooth and supersmooth distributions. When the distribution is ordinary smooth the condition (13) holds; it is not satisfied for supersmooth distributions.

An and $\mathrm{Hu}$ (20111) demonstrate that well-posedness holds automatically in a measurement error problem provided there is a mass at zero; this happens if there is a reporting error but with some non-zero probability some values 
are truthfully reported. Indeed if there is a mass at zero the error distribution is a mixture with the delta-function that has Fourier transform equal to 1, then $\phi$ is separated from zero and condition (13) for $\phi^{-1}$ holds.

A further extension is possible to deconvolution where the generalized function $\gamma$ is not necessarily a continuous function and can be singular. Then additional differentiability conditions are needed to ensure that $\gamma$ and $\phi$ are in a product pair. Let $\Gamma=S_{l, m}^{*}(V)$ with $l=\left(l_{1}, \ldots, l_{d}\right) ; \phi$ be a bounded continuous function that has continuous derivatives of any order $\leq l_{1}+\ldots+l_{d}$; then a product $\varepsilon=\gamma \phi$ exists in $S^{*}$ for any $\gamma \in \Gamma$.

Theorem 4 Assume $\gamma, \gamma_{n} \in S_{l, m}^{*}(V)$, and $\phi$ is a continuous regular function that has continuous derivatives $\partial^{l_{i}}$ for all subvectors $l_{i}$ of $l ; \varepsilon=\gamma \phi, \varepsilon_{n}=\gamma_{n} \phi$ for $n=1,2, \ldots ;$ and $\varepsilon_{n}-\varepsilon \rightarrow 0$ in $S^{*}$. If $\phi^{-1}$ and all $\partial^{l_{i}}\left(\phi^{-1}\right)$ for any subvector $l_{i}$ of each $l$ satisfy (13) then $\varepsilon_{n} \phi^{-1}$ exists in $S^{*}$ and $F t^{-1}\left(\varepsilon_{n} \phi^{-1}\right) \rightarrow g$ in $S^{*}$.

Proof. Denote by $c_{n}$ and $c$ the continuous functions for which $\varepsilon_{n}$ and $\varepsilon$ can be defined via the operator $\partial^{l}$. Consider the functional $\left(\phi^{-1} \varepsilon_{n}-\phi^{-1} \varepsilon, \psi\right)=$ $\left(\phi^{-1} \partial^{l}\left(c_{n}-c\right), \psi\right)$; it can be extended to the functional

$$
\left(c_{n}-c,(-1)^{|l|} \partial^{l}\left(\phi^{-1} \psi\right)\right)=(-1)^{|l|} \sum_{\left(l_{1} ; l_{2}\right)=l} \kappa\left(l_{1}, l_{2}\right)\left(c_{n}-c, \partial^{l_{1}}\left(\phi^{-1}\right) \partial^{l_{2}} \psi\right)
$$

where $\kappa\left(l_{1}, l_{2}\right)$ is the corresponding integer coefficient arising from differentiation of the product. All the functionals $\left(c_{n}-c, \partial^{l_{1}}\left(\phi^{-1}\right) \partial^{l_{2}} \psi\right)$ that enter into the linear combinations on the right-hand side satisfy the conditions of 
Lemma 2 and thus converge to zero. Therefore, $\gamma_{n}-\gamma=\left(\varepsilon_{n}-\varepsilon\right) \phi^{-1} \rightarrow 0$ in $S^{*}$.

By continuity in $S^{*}$ of the inverse Fourier transform the limit

$$
F t^{-1}\left(\varepsilon_{n} \phi^{-1}\right) \rightarrow g
$$

in $S^{*}$ follows.

If the measurement error model holds conditionally on some $d_{c}$-dimensional $x_{c}$ assume that the all the distribution functions are defined on $R^{d} \times R^{d_{c}}$ as $F\left(\cdot, x_{c}\right)$. For any generalized function $b$ on $R^{d_{x}} \times R^{d_{c}}$ the partial Fourier transform $F t_{x_{c}}$ is defined as follows: for $\psi\left(x, x_{c}\right) \in S$ with $x \in R^{d_{x}}, x_{c} \in R^{d_{c}}$ define $F t_{\mid x_{c}}(\psi)\left(s, x_{c}\right) \in S$ by $\int e^{i x^{T} s} \psi\left(x, x_{c}\right) d x$, then $\left(F t_{\mid x_{c}} b, \psi\right)=\left(b, F t_{\mid x_{c}}(\psi)\right)$. Consider the following possibilities: 1 . the generalized functions $\gamma, \phi, \varepsilon$ denote now the partial Fourier transforms or the distribution functions; 2. instead of distribution functions consider generalized density functions and denote by $\gamma, \phi, \varepsilon$ the corresponding partial Fourier transforms; 3. assume continuous differentiability of the probability distribution functions with respect to $x_{c}$ and consider conditional probability or, correspondingly, conditional generalized densities; additional conditions will be needed to make sure that dividing by the density of $x_{c}$ is possible in $S^{*}$. In all these cases (15) holds. To avoid imposing extra constraints here examine case 2. For deconvolution $\phi$ is assumed known and often $\phi$ does not vary with $x_{c}$. The conditions of Lemma 2 may not apply as the functions $\varepsilon, \varepsilon_{n}$ may no longer be continuous, 
however, since a probability distribution is a bounded monotone function, $\varepsilon$ is in some set $S_{1, d_{c}}^{*}(V)$ and thus theorem 4 requires only that all $\varepsilon_{n}$ belong to $S_{1, d_{c}}^{*}(V)$, that (13) is satisfied for $\phi^{-1}$ and that $\phi$ be differentiable in components of $x$.

The condition (13) for $\phi^{-1}$ is necessary for well-posedness: the example below shows that even in the weak topology of generalized functions wellposedness does not hold if it is not satisfied e.g. for deconvolution with supersmooth distributions.

Example. Consider the function $\phi(x)=e^{-x^{2}}, x \in R$, then $\phi^{-1}$ does not satisfy (13). Then there exists a sequence of functions $\gamma_{n}$ and a $\gamma$ in $S^{*}$ such that $\gamma_{n} \phi \rightarrow \gamma \phi$ in $S^{*}$, but $\gamma_{n}$ does not converge to $\gamma$ in $S^{*}$.

Define the function $b_{n}(x)=\phi^{-1}(x) I\left(n-\frac{1}{n}, n+\frac{1}{n}\right) ;$ then $b_{n} \in S^{*}$. Then define $\gamma_{n}=\gamma+b_{n}$ for some fixed $\gamma \in S^{*}$. Define $\varepsilon_{n}=\gamma_{n} \phi$ and $\varepsilon=\gamma \phi$; since $\phi \in S$, the products are always defined. Consider the difference $\varepsilon_{n}-\varepsilon$; it equals $I\left(n-\frac{1}{n}, n+\frac{1}{n}\right)$. In $S^{*}$ this sequence converges to zero. On the other hand, $\left(\gamma_{n}-\gamma, \psi\right)$ for any $\psi \in S$ equals

$$
\int_{n-2 / n}^{n+2 / n} e^{x^{2}} \psi(x) d x .
$$

Select $\psi \in S$ given by $\psi(x)=\exp (-|x|)$; then $\left(\gamma_{n}-\gamma, \psi\right)=$

$$
\begin{aligned}
\int_{n-2 / n}^{n+2 / n} e^{x^{2}} \psi(x) d x & \geq \int_{n-1 / n}^{n+1 / n} e^{x^{2}-x} d x \\
& \geq \frac{1}{2 n} e^{-\left(n+\frac{1}{n}\right)+\left(n-\frac{1}{n}\right)^{2}}
\end{aligned}
$$


Thus for this $\psi$ the values of the functional, $\left(\gamma_{n}-\gamma, \psi\right)$ diverge as $n \rightarrow \infty$, and so $\gamma_{n}-\gamma$ does not converge to zero in $S^{*}$

Note that since any $\psi \in D$ has bounded support in the example convergence of $\gamma_{n}$ to $\gamma$ holds in $D^{*}$.

\subsection{Well-posedness in $S^{*}$ of the solution to the system of equations (16)}

Recall (13) and define a subclass of functions in $S^{*}, \Phi(m, V)$, where $b \in$ $\Phi(m, V)$ if $b$ satisfies the condition

$$
\left(\left(1+t^{2}\right)^{-1}\right)^{m}|b(t)|<V<\infty
$$

The requirement that $b$ belong to $\Phi(m, V)$ implies that (13) applies uniformly in this class and also defines a bounded set in $S^{*}$. The next Theorem considers well-posedness of the problem in (3) (and correspondingly, (16)) under the identification conditions of Theorem 3(b). The conditions of Theorem 3(a) can be similarly considered.

Theorem 5 Suppose that $\left(\gamma_{n}, \phi_{n}\right), n=1,2, \ldots$ and $(\gamma, \phi)$ belong to a product pair $(\Gamma, \Phi)$. Additionally, let the conditions of Theorem 3(b) apply to each pair $\left(\gamma_{n}, \phi_{n}\right)$. Suppose that $\varepsilon_{1 n}-\varepsilon_{1} \rightarrow 0$ and $\varepsilon_{2 k n}-\varepsilon_{2 k} \rightarrow 0$ in $S^{*} ;$ the functions $\phi, \phi_{n}$ as well as $\phi^{-1}, \phi_{n}^{-1}$ restricted to $W$ all belong to some $\Phi(m, V)$ Then if

(a) $(\Gamma, \Phi) \equiv\left(S^{*}, O_{M}\right)$,

or 
(b) all $\varkappa_{k n}=\left(\phi_{n}\right)_{k}^{\prime} \phi_{n}^{-1}$, and $\varkappa_{k}=(\phi)_{k}^{\prime} \phi^{-1}$ are such that $\left(\varkappa_{k n}, \varepsilon_{1 n}\right)$, $\left(\varkappa_{k}, \varepsilon_{1}\right)$ belong to some product pair, the products $\varepsilon_{n} \phi_{n}^{-1}$ exist in $S^{*}$ and $F t^{-1}\left(\varepsilon_{1 n} \phi_{n}^{-1}\right) \rightarrow g$ in $S^{*}$.

Proof. (a) We have that $\phi, \phi_{n} \in \mathcal{O}_{M}$. It follows that $(\phi)_{k}^{\prime},\left(\phi_{n}\right)_{k}^{\prime} \in \mathcal{O}_{M}$. Also $(\phi)_{k}^{\prime},\left(\phi_{n}\right)_{k}^{\prime} \in \Phi\left(m^{\prime}, V\right)$, where $m^{\prime}=m+\iota$, with $\iota$ a vector of ones. From Theorem 3 it follows that for every $n$ the functions $\gamma_{n}$ and $\phi_{n}$ are uniquely identified on $W$. From now on we consider all functions and function spaces restricted to $W$, even when $W$ does not coincide with $R^{d}$, but keep the same notation. The functions belong also to $\Phi(\tilde{m}, V)$ where $\tilde{m}=m+m^{\prime}$. Without loss of generality assume that each $\varkappa_{k}$ is also in the same $\Phi(\tilde{m}, V)$, and so all $\varkappa_{k n}, \varkappa_{k}$ are in a bounded set in $S^{*}$. Since from condition $(a)$ it follows that $\varkappa_{k n}=\left(\phi_{n}\right)_{k}^{\prime} \phi_{n}^{-1} \in \mathcal{O}_{M}$, products are defined and from equations $\varepsilon_{1 n} \varkappa_{k n}-$ $\left(\left(\varepsilon_{1 n}\right)_{k}^{\prime}-i \varepsilon_{2 k n}\right)=0$ and convergence of $\varepsilon_{i n}$ to $\varepsilon_{i}$ we get that $\varepsilon_{1 n} \varkappa_{k n}-\varepsilon_{1} \varkappa_{k}$ converges to zero in $S^{*}$. For functions in $\mathcal{O}_{M}$ products with any elements from $S^{*}$ exist, thus $\varepsilon_{1 n} \varkappa_{k n}-\varepsilon_{1} \varkappa_{k n}$ exists; moreover $\left(\varepsilon_{1 n}-\varepsilon_{1}\right) \varkappa_{k n}$ converges to zero in $S^{*}$ by the hypocontinuity property (Schwartz, p.246). It follows that $\varepsilon_{1}\left(\varkappa_{k n}-\varkappa_{k}\right)$ converges to zero in $S^{*}$. Since $\varepsilon_{1}$ is supported on $W$ and $\left(\varkappa_{k n}-\varkappa_{k}\right) \in \mathcal{O}_{M}$ by continuity of the functional $\varepsilon_{1}$ it follows that $\varkappa_{k n}-\varkappa_{k}$ converges to zero on $W$. It then follows that $\phi_{n}-\phi \rightarrow 0$ in $S^{*}$ as well as pointwise and uniformly on bounded sets in $W$, the product $\phi^{-1} \phi_{n}^{-1}$ is in a bounded set in $S^{*}$, thus $\phi_{n}^{-1}-\phi^{-1}=\phi^{-1} \phi_{n}^{-1}\left(\phi-\phi_{n}\right)$ converges to zero in $S^{*}$.

Consider $\varepsilon_{1 n} \phi_{n}^{-1}-\varepsilon_{1} \phi^{-1}=\varepsilon_{1 n}\left(\phi_{n}^{-1}-\phi^{-1}\right)+\left(\varepsilon_{1 n}-\varepsilon_{1}\right) \phi^{-1}$; this difference 
converges to zero in $S^{*}$, thus $\gamma_{n}$ converges to $\gamma$ in $S^{*}$ and since the Fourier transform is continuously invertible in $S^{*}, g_{n}=F t^{-1}\left(\varepsilon_{1 n} \phi_{n}^{-1}\right) \rightarrow g$ in $S^{*}$.

(b) Modify the proof of (a) by stating existence of products based on the assumption in (b) rather than assuming the specific product pair $\left(S^{*}, O_{M}\right)$.

Thus well-posedness obtains for the solution to the errors in variables regression with Berkson type instruments that provides (3) as long as $\phi^{-1}$ and all the $\phi_{n}^{-1}$ are all in the same class $\left.\Phi(m, V)\right)$; e.g. a set of the characteristic functions would satisfy this if they are not supersmooth and the growth of all $\phi_{n}^{-1}$ is bounded by the same order polynomial. With the condition (a) which does not restrict $\gamma \in S^{*}$ the Theorem will hold if the supports of $\phi, \phi_{n}$ are bounded or if these were Fourier transforms of functions with some singularity points (e.g. from distributions with mass points), when $\phi$ would include a constant. The condition (b) would require imposing restrictions on degree of singularity of $\gamma$ and further differentiability conditions on $\phi$ and $\phi_{n}$ of the type imposed in Theorem 4 to ensure that all the products exist. Even if in the model $\phi$ is a characteristic function, $\phi_{n}$ need not necessarily be characteristic functions, but just continuous functions that satisfy the conditions. This result did not require any restrictions on $\gamma$ and thus on the regression function in the model beyond belonging to $S^{*}$. Under Theorem 3(a) a similar result holds after imposing the conditions on $\gamma, \gamma_{n}$ rather than $\phi, \phi_{n}$ to ensure that generalized functions $\varkappa_{k n}=\left(\gamma_{n}\right)_{k}^{\prime} \gamma_{n}^{-1}$ belong to $\mathcal{O}_{M}$ and to some $\Phi(\tilde{m}, V)$. 
For example, if as in Cunha et al (2010) both $\gamma$ and $\phi$ are characteristic functions and thus continuous and bounded, products exist and Theorem 5(b) applies, as long as the conditions of Theorem 3 are satisfied and the condition (21) is satisfied for the inverses of the functions in the sequence,

that is e.g. under Theorem 3(a) for $\gamma_{n}^{-1}$, or under 3(b) for $\phi_{n}^{-1}$, so that the corresponding distributions are not supersmooth and the polynomial lower bound holds uniformly, well-posedness holds.

The implications that well-posedness has for estimation are two-fold. One is that unless well-posedness holds, that is the inverse mapping from the class of the known functions into the class of identified solutions is continuous, the solutions corresponding to the consistently estimated known functions will not in general provide consistent estimators for the solutions. The other is that in a well-posed problem consistent estimation of the known functions automatically gives rise to consistency of plug-in estimators of solutions; the next section examines consistent estimation of the deconvolution and the solution to the system (3) in the space $S^{*}$.

\section{Random generalized functions and stochas- tic convergence}

This section examines stochastic convergence of the solutions to the deconvolution equation (21) and to (3), equivalently to (15) and (16). If some 
consistent estimators are available for either the function $w\left(w_{1}\right.$ and $\left.w_{2 k}\right)$ or, equivalently, for the Fourier transform, $\varepsilon\left(\varepsilon_{1}\right.$ and $\left.\varepsilon_{2 k}\right)$, stochastic convergence of the solutions provides consistency results for plug-in estimators of $g$ as long as well-posedness holds. Since generalized functions in any space $G^{*}$ are represented as linear continuous functionals on $G$ results for random functionals are applicable here, the specific feature is that for any generalized function $b \in G^{*}$ one needs to consider the collection of random functionals indexed by the functions from the space $G$.

\subsection{Random generalized functions}

Following Gel'fand and Vilenkin (1964) define random generalized functions as random linear continuous functionals on the space of test functions (see e.g. Koralov and Sinai, 2007 who consider specifically $S^{*}-$ ch.17). In particular,

any random generalized function $\tilde{b}$ on $G$ is represented by a collection of (complex-valued) random variables on a common probability space that are indexed by $\psi \in G$, denoted $(\tilde{b}, \psi)$, such that

(a) $\left(\tilde{b},\left(a_{1} \psi_{1}+a_{2} \psi_{2}\right)\right)=a_{1}\left(\tilde{b}, \psi_{1}\right)+a_{2}\left(\tilde{b}, \psi_{2}\right)$ a.s.;

(b) if $\psi_{k n} \rightarrow \psi_{k}$ in $S$ as $n \rightarrow \infty, k=1,2 . ., m$, then vectors

$$
\left(\left(\tilde{b}, \psi_{1 n}\right) \ldots\left(\tilde{b}, \psi_{m n}\right)\right) \rightarrow_{d}\left(\left(\tilde{b}, \psi_{1}\right) \ldots\left(\tilde{b}, \phi_{m}\right)\right)
$$

where $\rightarrow_{d}$ denotes convergence in distribution.

As shown in Gel'fand and Vilenkin, equivalently, there exists a probabil- 
ity measure on $G^{*}$ such that for any set $\psi_{1}, \ldots \psi_{m} \in G$ the random vectors $\left(\left(b, \psi_{1}\right), \ldots\left(b, \psi_{m}\right)\right)$ have the same distribution as for some random functional $\tilde{b},\left(\left(\tilde{b}, \psi_{1}\right) \ldots\left(\tilde{b}, \psi_{m}\right)\right)$. An example is a generalized Gaussian process $b$, so defined if for any $\psi_{1}, \ldots, \psi_{m}$ the joint distribution of $\left(b, \psi_{1}\right), \ldots,\left(b, \psi_{m}\right)$ is Gaussian. A generalized Gaussian process is uniquely determined by its mean functional, $\mu_{b}:\left(\mu_{b}, \psi\right)=E(b, \psi)$, and the covariance bilinear functional, $B_{b}\left(\psi_{i}, \psi_{j}\right)=E\left(\left(b, \psi_{i}\right)\left(b, \psi_{j}\right)\right)$.

Gelfand, Vilenkin (v.4, p. 260) give the covariance functional of the generalized derivative, $W^{\prime}$, of the Wiener process as

$$
B_{W^{\prime}}\left(\psi_{1}, \psi_{2}\right)=\int_{0}^{\infty} \psi_{1}(t) \overline{\psi_{2}(t)} d t
$$

where the overbar represents complex conjugation; for real-valued processes it is not needed. The Fourier transform of a Gaussian random process $b$ is also a Gaussian random process with covariance functional

$$
B_{F t(b)}\left(\psi_{i}, \psi_{j}\right)=E\left(\left(b, F t\left(\psi_{i}\right)\right)\left(b, F t\left(\psi_{j}\right)\right)\right) .
$$

So for $F t\left(W^{\prime}\right)$ the covariance functional is

$$
B_{F t\left(W^{\prime}\right)}\left(\psi_{1}, \psi_{2}\right)=\int_{0}^{\infty} F t\left(\psi_{1}\right)(\zeta) \overline{F t\left(\psi_{2}\right)(\zeta)} d \zeta .
$$

The mean functional is zero for $W^{\prime}$ and $F t\left(W^{\prime}\right)$.

Gelfand and Vilenkin (1964) provide definitions and results for general- 
ized random functions in $G^{*}=D^{*}$, rather than $S^{*}$. One can similarly define random generalized functions on other spaces of test functions, not necessarily infinitely differentiable, e.g. on $D_{k}$ of $k$ times continuously differentiable functions with compact support, leading to space $D_{k}^{*}$ of random linear continuous functionals on $D_{k}$.

\subsection{Stochastic convergence of random generalized func- tions}

A random sequence $b_{n}$ of elements of a space of generalized functions $G^{*}$ converges to zero in probability: $b_{n} \rightarrow_{p} 0$ in $G^{*}$, or almost surely: $b_{n} \rightarrow_{a . s .} 0$ in $G^{*}$ if for any finite set $\psi_{1}, \ldots \psi_{v} \in G$ the random vectors $\left(\left(b_{n}, \psi_{1}\right), \ldots\left(b_{n}, \psi_{v}\right)\right) \rightarrow_{p}$ $(0, \ldots, 0)$ or correspondingly, $\operatorname{Pr}\left(\left(\left(b_{n}, \psi_{1}\right), \ldots\left(b_{n}, \psi_{v}\right)\right) \rightarrow(0, \ldots, 0)\right)=1$.

Similarly, convergence in distribution of generalized random processes $b_{n} \Rightarrow_{d} b$ is defined by the convergence of all multivariate distributions for random vectors $\left(\left(b_{n}, \psi_{1}\right), \ldots\left(b_{n}, \psi_{v}\right)\right) \rightarrow_{d}\left(\left(b, \psi_{1}\right) \ldots\left(b, \psi_{v}\right)\right)$ for any set $\psi_{1}, \ldots, \psi_{v} \in$ $G$.

Remark 1. (a) If $b_{n}-b \rightarrow_{p} 0$ in $S^{*}$ then $F t\left(b_{n}\right)-F t(b) \rightarrow_{p} 0$ in $S^{*}$ and $F t^{-1}\left(b_{n}\right)-F t^{-1}(b) \rightarrow_{p} 0$ in $S^{*}$. Indeed, for any set $\psi_{1}, \ldots \psi_{v} \in S$

$$
\begin{aligned}
& \left(\left(F t\left(b_{n}\right)-F t(b), \psi_{1}\right), \ldots,\left(F t\left(b_{n}\right)-F t(b), \psi_{v}\right)\right) \\
= & \left(\left(b_{n}-b, F t\left(\psi_{1}\right)\right), \ldots,\left(b_{n}-b, F t\left(\psi_{v}\right)\right)\right)
\end{aligned}
$$

and since the set $F t\left(\psi_{1}\right), \ldots, F t\left(\psi_{v}\right) \in S$ then $\left(\left(b_{n}-b, F t\left(\psi_{1}\right)\right), \ldots,\left(b_{n}-\right.\right.$ 
b, Ft $\left.\left.\left(\psi_{v}\right)\right)\right) \rightarrow_{p}$ 0. Similarly for $F t^{-1}$.

(b) If $\mu \in G$ and $b_{n}-b \rightarrow_{p} 0$ in $G^{*}$, then $b_{n} \mu-b \mu \rightarrow_{p} 0$ in $G^{*}$. This follows similarly from the fact that $\mu \psi \in G$ for $\psi \in G$.

(c) Parts (a) and (b) of this Remark also hold with $\rightarrow_{a . s .}$ replacing $\rightarrow_{p}$ and with convergence to zero replaced by convergence in distribution to a limit generalized random process.

\section{Consistent estimation of solutions to stochas- tic equations}

Suppose that the known functions, $w$ or $w_{1}, w_{2 k}$ (equivalently, $\varepsilon$ or $\varepsilon_{1}, \varepsilon_{2 k}$ ) are consistently estimated in $S^{*}$. In the models discussed here these functions are density functions or conditional mean functions; typically these are ordinary functions for which commonly used nonparametric estimators are shown to be consistent pointwise or in some norm (uniform or $L_{1}$, say) under some sufficient conditions. If the functions and the estimators can be represented as elements in $S^{*}$ then convergence in many common norms implies convergence in the weaker topology of $S^{*}$, so that such consistent estimators are consistent in $S^{*}$ and the following discussion of consistency of the solutions to convolution equations considered here applies. However, consistency in $S^{*}$ applies more widely than the usual consistency results. For example, as shown in Zinde-Walsh, 2008, kernel estimators of density converge as stochastic generalized functions even when they diverge pointwise (e.g. at 
mass points, or for fractal measures).

For the equations considered here, when identification and well-posedness holds consistent estimation of the known functions can provide consistent plug-in estimators for the solutions as long as the estimators all be in the classes over which well-posedness obtains.

The fact that well-posedness implies consistency of some plug-in estimators holds generally. Denote (generically) the known functions by $w_{1}, \ldots w_{M}$, their consistent in topology of $S^{*}$ estimators by $\hat{w}_{i}$; denote the solutions by $g_{1}, \ldots, g_{K}$ and the plug-in estimators from solving the equations using $\hat{w}_{i}$ by $\hat{g}_{j}$. If the estimators, $\hat{w}_{i}$, are in classes where well-posedness holds, then for any set of $\left(\psi_{1}, \ldots \psi_{v}\right)$ from $S$ and any neighborhood $N_{v K}(0)$ of zero in real or complex $v K$ - dimensional Euclidean space there is a neighborhood of zero, $\tilde{N}_{v M}(0)$ in the corresponding $v M$-dimensional space such that event $E_{w}=\left\{\left(\left(\hat{w}_{1}, \psi_{1}\right), \ldots,\left(\hat{w}_{1}, \psi_{v}\right),\left(\hat{w}_{2}, \psi_{1}\right), \ldots,\left(\hat{w}_{M}, \psi_{v}\right)\right)^{\prime} \in \tilde{N}_{v M}(0)\right\}$ by well-posedness implies the event

$$
E_{g}=\left\{\left(\left(\hat{g}_{1}, \psi_{1}\right), \ldots,\left(\hat{g}_{1}, \psi_{v}\right),\left(\hat{g}_{2}, \psi_{1}\right), \ldots,\left(\hat{g}_{K}, \psi_{v}\right)\right)^{\prime} \in N_{v K}(0)\right\}
$$

Consistency of $\hat{w}_{i}$ as $n \rightarrow \infty$ means that for large enough $n$ probability of $E_{w}$ can be arbitrarily close to 1 , thus probability of $E_{g}$ is as close or closer to 1 . Thus the condition for consistent plug-in estimation is that the estimators are in the classes of generalized functions that provide well-posedness of solutions with probability approaching 1 . 


\subsection{Consistency of deconvolution: solving (15)}

The well-posedness condition directly applies to the measurement error deconvolution problem where $\phi$ is known and the conditions apply only to estimators of observables. The requirement of boundedness of the sequence of the estimators in the space $S^{*}$ would follow if the norms of the functions were bounded in probability; $L_{p}$ are the spaces typically used in the literature (e.g. Fan, 1991; Carrasco and Florens, 2010). But here more generally consistency of plug-in deconvolution holds for any probability distribution; the only important restriction is on $\phi^{-1}$ to satisfy (13) ; that is for the measurement error not to be supersmooth.

Moreover, the functions in (2) and the corresponding (15) need not be generalized densities and characteristic functions; the conditions for consistent estimation follow from those for well-posedness and are essentially that the two functions belong to a convolution pair (equivalently, Fourier transforms to a product pair) and that the known function be continuous and $\phi^{-1}$ satisfy (13) .

For example, consider in the univariate case a generalized density function, $w$. One can use empirical distribution functions to estimate the corresponding distribution function, then the estimator of generalized density by generalized derivatives provides as an estimator the sum of delta-functions: $\hat{w}_{n}=\frac{1}{n} \sum_{j=1}^{n} \delta\left(x_{j}\right)$, where $\left(\delta\left(x_{j}\right), \psi\right)=\psi\left(x_{j}\right)$. Then the corresponding Fourier transform $\hat{\varepsilon}_{n}=F t\left(\hat{w}_{n}\right)$ is given by a continuous function $\hat{\varepsilon}_{n}(s)=\frac{1}{n} \sum_{j=1}^{n} e^{i s^{T} x_{j}}$. 
Deconvolution using the deconvolution kernel (as e.g. in $\mathrm{An}$ and $\mathrm{Hu}, 2012$ ) is applied when the density of the mismeasured variable is assumed to exist and belong in some $L_{p}$, the deconvolution kernel incorporates spectral cut-off by employing estimators of the form $\tilde{\varepsilon}_{n}=\hat{\varepsilon}_{n}(s) I\left(|s|<T_{n}\right)$, where $T_{n}$ goes to $\infty$ as $n \rightarrow \infty$ at some rate; the indicator function allows for smoothing in the inverse Fourier transform.

By the results here consistency in $S^{*}$ is ensured for both estimators. Indeed since empirical distribution converges uniformly in probability to the distribution function, it thus converges in $S^{*}$, then the generalized derivatives also converge in $S^{*}$ and by continuity of the Fourier transform estimators $\hat{\varepsilon}_{n}$ and for $T_{n} \rightarrow \infty$ also $\tilde{\varepsilon}_{n}$ converge to $\varepsilon$ in probability. All these functions are continuous and bounded and only (13) for $\phi^{-1}$ is needed for consistency of the deconvolution solution in $S^{*}$ and the spectral cut-off is not required. The following remark summarizes this result.

Remark 2. If the functions in equation (2) are all generalized density functions, the known characteristic function $\phi$ is such that $\phi^{-1}$ satisfies (13), $\left\{z_{i}\right\}_{i=1}^{n}$ is a random sample from the distribution with the generalized density $w$, the deconvolution estimator

$$
\hat{g}_{n}=F t^{-1}\left(\phi^{-1} \hat{\varepsilon}_{n}\right) \text { where } \hat{\varepsilon}_{n}(s)=\frac{1}{n} \sum_{j=1}^{n} e^{i s^{T} z_{j}}
$$

is consistent in $S^{*}$ : for any finite set $\psi_{1}, \ldots \psi_{v} \in S$ the random vector $\left(\left(\hat{g}_{n}-g, \psi_{1}\right), \ldots,\left(\hat{g}_{n}-g, \psi_{v}\right)\right)^{T} \rightarrow_{p} 0$. 
Regularization with suitable spectral cut-off in the case of supersmooth error distributions typically provides a sequence of estimators that will converge in norm, albeit slowly for supersmooth distributions (Fan, 1991); convergence to the limit in the normed space implies convergence in the topology of $S^{*}$. However, if $\phi$ is not supersmooth the class of generalized functions where consistent estimation holds does not include all probability distributions. Indeed spectral cut-off estimation provides generalized densities that are a limit of inverse Fourier transforms of truncated generalized functions. Schwartz (1964, pp.271-273) gives a characterization of any function in $S^{*}$ with Fourier transform that has compact support (in a cube $\left|z_{k}\right|<C, k=1, \ldots d$ ) based on Wiener-Paley theorem. Such a function is a continuous function $g$ that can be extended to a entire analytic function $G$ of a complex argument and is of exponential type $\leq 2 C$, meaning

$$
\lim _{|z| \rightarrow \infty} \frac{\log |G(z)|}{\left|z_{1}\right|+\ldots+\left|z_{d}\right|} \leq 2 C .
$$

Thus as long as $g$ is such a function or a limit of such functions it can be expressed via the regularized solution. As in Schwartz the subspace of all functions of exponential type (for any finite C) can also be considered. However, if $g$ does not belong to this subspace regularized solutions may not converge to it even in $S^{*}$. 


\subsection{Consistency of estimated solutions to the system of equations (3) and (16)}

Establishing consistency for the system of equations is more complicated since consistency requires not only conditions on estimators of known functions of the observables, $\varepsilon$., but also verification that the resulting estimators of $\phi$ satisfy the conditions of well-posedness in Theorem 5 .

The next Theorem gives conditions for a consistent plug-in nonparametric estimator for the model $(7-9)$ that leads to the system (3) and thus (16) with continuously differentiable $\phi$.

Semiparametric generalized method of moments estimation of this model for a class of regression functions that includes functions in $L_{1}\left(R^{d}\right)$ was proposed by Wang and Hsiao (2010); semiparametric estimation was also discussed for somewhat different classes of univariate parametric regression functions in Schennach (2007) and Zinde-Walsh (2009). Semiparametric estimation of polynomial regression is in Hausman, Newey, Ichimura and Powell (1991).

Start by formally stating the assumptions.

Assumption 4 (model). In the model (7-9) in $R^{d}$ the moments of the errors $E\left(u_{y} \mid z, u, u_{x}\right)=0 ; E\left(u_{x} \mid z, v\right)=0 ; z$ is independent of $u ; g$ is a generalized function in $S^{*}$.

This assumption implies that if the function $g$ is a regular locally summable function it cannot grow at infinity at a rate faster than some polynomial rate. 
This assumption does not exclude the possibility that $g$ has some singularities, for example, it could be a sum of $\delta$-functions or a mix of a regular function and some peaks represented by $\delta$-functions.

Denote by $f_{z}$ the density of $z$. Recall that $f$ denoted the generalized density of $u$ and $\gamma=F t(g) ; \phi=F t(f), \varepsilon .=F t(w$.$) .$

\section{Assumption 5 (support).}

(a) $\operatorname{supp}(\gamma)$ is a connected set that includes 0 as an interior point;

(b) $\operatorname{supp}(\phi)=R^{d}$.

$$
\text { (c) } \operatorname{supp}\left(f_{z}\right)=R^{d} \text {. }
$$

Assumption 5(a) would not be satisfied by a polynomial regression function when support of $\gamma$ consists of one point 0 ; semiparametric estimation for that case was provided in Hausman et al (1991). Support assumptions (b) and (c) are standard; they could be relaxed.

Recall that here $\phi$ is a characteristic function; $\phi(0)=1$.

\section{Assumption 6 (generalized functions).}

(a) The function $f_{z}$ is continuous and $\phi$ belongs to $O_{M}$.

(b) The continuous functions $w$. belong to a bounded set in $S^{*}, S_{0, m}^{*}(V)$; also, $\phi_{k}^{\prime}$ for $k=1, \ldots, d$, as well as $\phi^{-1}$ and $f_{x}^{-1}$ all belong to $S_{0, m}^{*}(V)$.

(c) The regression function $g$ is absolutely integrable.

(c') The regression function $g$ can be represented as a sum $g=g_{L 1}+g_{g}$, where $g_{L 1}$ is absolutely integrable and $g_{g}$ is such that the Fourier transform of $g_{g}$ in $S^{*}, \gamma_{g}=F t\left(g_{g}\right)$, is singular and thus has support set $\Lambda_{g}$ that is compact and of zero Lebesgue measure; $\Lambda_{g}$ is a proper subset of $\operatorname{supp}(\gamma)$. 
The generalized function $\gamma_{g}$ is such that there exists a deterministic sequence of regular functions, $\left(\gamma_{g}\right)_{n}$ that converges to $\gamma_{g}$ in $S^{*}$ and such that support of $\left(\gamma_{g}\right)_{n}$ is in a compact set $\Lambda_{g n}$; there exists $\zeta_{0}>0$ such that $\left|\left(\gamma_{g}\right)_{n}\right|>2 \zeta_{0}$ and for $\gamma_{L 1}=F t\left(g_{L 1}\right)$ on $\Lambda_{g}\left|\gamma_{L 1}-\left(\gamma_{g}\right)_{n}\right|>\zeta_{0}$.

Assumptions 6(a) and 6(b) imply that $w$. can be divided by $f_{z}$ and any generalized function can be divided by $\phi$. Requiring that $\phi$ be in $O_{M}$ is sufficient to ensure that the model leads to equations (3) in $S^{*}$. More detailed conditions similar to those employed in Theorem 2 would allow relaxing the infinite differentiability assumption 6(a). In particular, if $\gamma$ is a characteristic function Assumption 6(a) for $\phi$ is not needed.

Continuity of $w$. in 6(b) would follow by properties of convolution if either $g$ were continuously differentiable, or $f$ were continuous.

In (b) using the same bound on growth, $m$, and the same $V$ for all the functions simplifies exposition without loss of generality. The bounds could be liberal but are assumed known in the construction of estimators. The constraint on the $\phi^{-1}$ restricts the measurement error from being supersmooth and the constraint on $f_{z}^{-1}$ does not permit fast decline to zero at infinity for the density of conditioning $z$; these would be automatically satisfied if supports were bounded.

Assumption (c) implies that $\gamma$ and therefore $\varepsilon_{1}$ are continuous functions. Indeed an integrable function has a continuous Fourier transform, $\gamma$, and $\varepsilon_{1}=\gamma \phi$ is continuous since $\phi$ is a characteristic function and thus continuous.

Assumption 6(c') holds more generally, e.g. if $g$ is a sum of an integrable 
function and a polynomial or a sin or cos function; in such cases $\gamma$ is a sum of a continuous function (that is separated from zero on bounded sets within its support) with singular functions such as the $\delta$-function, shifted $\delta$-function and its derivatives. The support conditions in the assumption imply that the open support set of the continuous $\gamma_{L 1}$ contains $\Lambda_{g}$. The existence of a function sequence converging in $S^{*}$ to $\gamma_{g}$ with the support properties stated follows from the general properties of generalized functions; the only substantive condition there is that the values of the approximating functions $\left(\gamma_{g}\right)_{n}$ be separated from zero and from the values of $\gamma_{L 1}$. For example, if $g$ included an additive constant, then $\gamma_{g}$ is a $\delta$-function, and $\left(\gamma_{g}\right)_{n}$ could be selected as a sequence of step functions. These approximating functions need not be specified, only their existence is required for the proof.

The next assumption is on the stochastic properties of the data generating process, the sampling and on the kernel and bandwidth.

Assumption 7. Moments of order $q>d+1$ of $\frac{1}{\left(1+z^{2}\right)^{m}} y, \frac{1}{\left(1+z^{2}\right)^{m}} x y$ conditional on $z$ are bounded; $\left\{x_{i}, y_{i}, z_{i}\right\}_{i=1}^{n}$ is a random sample from $\{x, y, z\}$; the kernel $K$ is the indicator function of the unit sphere; the bandwidth $h$ is such that $h \rightarrow 0$ and satisfies $n^{1-\frac{d}{q-1}} h^{d} \rightarrow \infty$.

Note that Assumption 6(b) implies boundedness of first conditional moments (functions $w$.) of $\frac{1}{\left(1+z^{2}\right)^{m}} y, \frac{1}{\left(1+z^{2}\right)^{m}} x y$.

Define the estimators (denoting by $\left(x_{k}\right)_{i}$ the $i t h$ observation on the $k$ th 
component of vector $x$ )

$$
\begin{aligned}
\hat{w}_{1}(z)_{n}= & \frac{\sum_{i=1}^{n} y_{i} K\left(\frac{z_{i}-z}{h}\right)}{\sum_{i=1}^{n} K\left(\frac{z_{i}-z}{h}\right)} ; \\
\hat{w}_{2 k}(z)_{n}= & \frac{\sum_{i=1}^{n} y_{i}\left(x_{k}\right)_{i} K\left(\frac{z_{i}-z}{h}\right)}{\sum_{i=1}^{n} K\left(\frac{z_{i}-z}{h}\right)}, k=1, \ldots d .
\end{aligned}
$$

By assumption 6(b) the functions $\frac{1}{\left(1+z^{2}\right)^{m}} w_{1}(z), \frac{1}{\left(1+z^{2}\right)^{m}} w_{2 k}(z)$ are bounded in absolute value by $V$; by $6\left(\right.$ a) the density of $z, f_{z}(z)$, exists and is continuous, then that for $z$ in any closed sphere $S(x, r) \in \operatorname{supp}\left(f_{z}\right)$ the essinf $f_{z}(z)>$ 0. Together with Assumption 7 this is sufficient to ensure that estimators $\frac{1}{\left(1+z^{2}\right)^{m}} \hat{w}_{1}(z)_{n}, \frac{1}{\left(1+z^{2}\right)^{m}} \hat{w}_{2 k}(z)_{n}$, of $\frac{1}{\left(1+z^{2}\right)^{m}} w_{1}(z), \frac{1}{\left(1+z^{2}\right)^{m}} w_{2 k}(z)$, with $\hat{w} \cdot(z)_{n}$ computed as (22), with $K$ and $h$ that satisfy assumption 7 converge in probability uniformly over any compact set (Devroye, 1978). If $m=0$ the moment condition is a usual condition made for the Nadaraya-Watson estimator of $w$; here essentially just the growth of the conditional moment functions has to be restricted; this provides estimators that converge in the topology of $S^{*}$. The bound $V$ is assumed known here; more restrictive assumptions, including in particular differentiability of $w$. could provide a uniform over compact sets rate of convergence for e.g. asymptotically optimal estimators (e.g. Stone, 1982); then $V$ that defines $\tilde{w}_{\cdot n}$ could grow with sample size.

Uniform convergence in probability implies that the estimators converge in probability in the topology of $D^{*}$. If the functions have compact support 
this implies convergence in $S^{*}$ since on compact support it coincides with convergence in $D^{*}$, but on unbounded support growth at infinity needs to be controlled for convergence in $S^{*}$. Thus, for any generic estimator $\hat{w}_{n}$ represented by a regular function, $\hat{w}(z)_{n}$, of a regular generalized function $w=w(z)$ in $S_{1, m}^{*}(V)$ define a corresponding estimator $\tilde{w}_{n}=\tilde{w}(z)_{n}$ by setting it to $\hat{w}(z)_{n}$ if $\left|\hat{w}(z)_{n}\right|<V\left(1+z^{2}\right)^{m}$, and $V\left(1+z^{2}\right)^{m}$ otherwise.

Lemma 3. Suppose that the estimator $\hat{w}_{n}$ converges to $w$ in probability uniformly on bounded sets; then $\tilde{w}_{n}$ converges to $w$ in probability in $S^{*}$ and the corresponding Fourier transform $\hat{\varepsilon}_{n}=F t\left(\tilde{w}_{n}\right)$ converges in probability in $S^{*}$ to $\varepsilon=F t(w)$.

Proof. Consider a set $\psi_{1}, \ldots, \psi_{v} \in S$. For any $\zeta>0$ find a compact set $\Lambda$ such that for $\tilde{\psi}$ representing any of $\psi_{1}, \ldots, \psi_{v}, F t\left(\psi_{1}\right), \ldots, F t\left(\psi_{v}\right)$

$$
\int_{R^{d} \backslash \Lambda} V\left(1+t^{2}\right)^{m}|\tilde{\psi}(t)| d t<\zeta
$$

Consider $\left|\tilde{w}_{n}-w\right| \leq\left|\hat{w}_{n}-w\right| I(z \in \Lambda)+\left|\tilde{w}_{n}-\hat{w}_{n}\right| I(z \in \Lambda)+\left|\tilde{w}_{n}-w\right| I\left(z \in R^{d} \backslash \Lambda\right)$. Then for any $\zeta_{1}$ there exists $N$ such that $\operatorname{Pr}\left(\left|\left(\tilde{w}_{n}-w, \tilde{\psi}\right)\right|>\zeta_{1}\right) \leq$ $\operatorname{Pr}\left(\sup _{\Lambda}\left|\hat{w}_{n}-w\right|\left|\int \tilde{\psi}(t) d t\right|>\zeta_{1}\right)+\operatorname{Pr}\left(z \in \Lambda:\left|\tilde{w}_{n}-\hat{w}_{n}\right|\left|\int \tilde{\psi}(t) d t\right|>\zeta_{1}-2 \zeta\right) \leq \zeta_{1}$, since the value $\left|\left(\tilde{w}_{n}-w, \tilde{\psi}\right)\right|$ outside $\Lambda$ is bonded with certainty by $2 \zeta$ and the two probabilities on bounded $\Lambda$ can be bounded because of uniform convergence in probability of the estimators. Recall that for Fourier transforms 
$\left.\left(F t\left(\tilde{w}_{n}-w\right), \psi\right)=\left(\hat{w}_{n}-\hat{w}, F t(\psi)\right)\right)$. Thus convergence in probability in $S^{*}$ of the estimators of the functions $\hat{w}$, and also of the Fourier transforms $\varepsilon$ is established.

Denote $\operatorname{Ft}\left(\tilde{w}_{\cdot n}\right)$ by $\hat{\varepsilon}_{\cdot n}$, note that by construction these random generalized functions are infinitely differentiable since by the assumption on the kernel $K$ the support of $\hat{w}_{\cdot n}$ is bounded and thus the Fourier transform is differentiable. The Theorem below establishes consistency of plug-in estimators.

Theorem 6 If Assumptions 4-7 are satisfied, then

(i) if $\operatorname{supp}(\gamma)$ is bounded the plug-in estimator

$$
\hat{\gamma}_{n}=\left[\exp \left(-\int_{0}^{s} \sum_{k=1}^{d} \hat{\varepsilon}_{1 n}^{-1}(t)\left(\left(\hat{\varepsilon}_{1 n}(t)\right)_{k}^{\prime}-i \hat{\varepsilon}_{2 k n}(t)\right) d t_{k}\right] \hat{\varepsilon}_{1 n}\right.
$$

is such that it exists with probability going to 1 and $F t^{-1}\left(\hat{\gamma}_{n}\right)-g \rightarrow_{p} 0$ in $S^{*}$;

(ii) generally the estimator $\hat{\gamma}_{n}=\tilde{\phi}^{-1} \hat{\varepsilon}_{1 n}$ with

$$
\tilde{\phi}^{-1}=\tilde{\phi}_{n}^{-1}(s)=\left[\exp \left(-\int_{0}^{s} \sum_{k=1}^{d} \hat{\varepsilon}_{1 n}^{-1}(t)\left(\left(\hat{\varepsilon}_{1 n}(t)\right)_{k}^{\prime}-i \hat{\varepsilon}_{2 k n}(t)\right) d t_{k}\right],\right.
$$

if

$$
\mid\left[\exp \left(-\int_{0}^{s} \sum_{k=1}^{d} \hat{\varepsilon}_{1 n}^{-1}(t)\left(\left(\hat{\varepsilon}_{1 n}(t)\right)_{k}^{\prime}-i \hat{\varepsilon}_{2 k n}(t)\right) d t_{k}\right] \mid<V\left(1+s^{2}\right)^{m}\right.
$$

and

$$
V\left(1+s^{2}\right)^{m}
$$


otherwise, is such that it exists with probability approaching 1 and $F t^{-1}\left(\hat{\gamma}_{n}\right)-$ $g \rightarrow{ }_{p} 0$ in $S^{*}$.

Proof. The proof consists of the following steps.

Step 1 considers a compact set inside the support of $\gamma$ and shows that on such a set consistency in $S^{*}$ follows.

Step 2 examines the continuous part of $\gamma, \gamma_{L 1}$, and convergence is probability of estimators defined on compact sets that exclude a set containing the support of the singular part.

Combining the results of Step 1 and 2 concludes the proof of (i).

Step 3 considers the case of unbounded support with $\tilde{\phi}_{n}^{-1}$ defined in (ii). On any compact set with this estimator replacing $\hat{\phi}_{n}^{-1}$ the results in Steps 1 and 2 hold so consistency on a bounded set obtains. Consistency on the unbounded support is shown in topology of $S^{*}$ by selecting for any set of $\psi_{1}, \ldots \psi_{v}$ from $S$ the corresponding compact set defined in Lemma 3 to bound all the functionals in probability outside of the compact set to prove (ii).

Next, the details are provided.

Step 1. First, $\hat{\varepsilon}_{1 n}$; this is a sequence of continuous functions that converge in probability in $S^{*}$ by Lemma 3 . Fourier transforms of functions in $L_{1}$ are uniformly continuous on compact sets. If Assumption 6(c) holds convergence is to the continuous function $\varepsilon_{1}$. Then $\hat{\varepsilon}_{1 n}$ converge in probability pointwise and uniformly on the compact set $\Lambda$. Then for any $0<\zeta_{1}, \zeta_{2}<1$ we can find 
$N_{1} \equiv N\left(\Lambda, \zeta_{1}, \zeta_{2}\right)$ such that for $n>N_{1}$

$$
\operatorname{Pr}\left(\sup _{\Lambda}\left|\hat{\varepsilon}_{1 n}-\varepsilon_{1}\right|>\zeta_{1}^{3}\right)<\zeta_{2}
$$

Consider $\Lambda$ that satisfies $\inf _{\Lambda}\left|\varepsilon_{1}\right|>0$. Set $\zeta_{1} \leq \inf _{\Lambda}\left|\varepsilon_{1}\right|$, then

$$
\begin{aligned}
& \operatorname{Pr}\left(\inf _{\Lambda}\left|\hat{\varepsilon}_{1 n}\right|\right.\left.<\zeta_{1}\right)<\zeta_{2} ; \text { and } \\
& \operatorname{Pr}\left(\sup _{\Lambda}\left|\hat{\varepsilon}_{1 n}^{-1}\right|>\zeta_{1}^{-1}\right)<\zeta_{2} .
\end{aligned}
$$

Under $6\left(c^{\prime}\right)$ for a singular $\gamma_{g}$ there exists a deterministic sequence of regular functions, $\left(\gamma_{g}\right)_{n}$ that converges to $\gamma_{g}$ in $S^{*}$ and such that support of $\left(\gamma_{g}\right)_{n}$ is in a compact set $\Lambda_{g n}$ that is a proper subset of support of $\gamma$; it can be selected such that for some sequence $\zeta_{g n} \rightarrow 0$ the Lebesgue measure of $\Lambda_{g n}, \lambda\left(\Lambda_{g n}\right)<\zeta_{g n} ; \Lambda_{g n_{1}} \subset \Lambda_{g n_{2}}$ for $n_{1}>n_{2}$ and on any $\Lambda_{g n}$ for some fixed $\zeta_{0}$ we get $\inf _{\Lambda_{g n}}\left|\left(\gamma_{g}\right)_{n}\right|>\zeta_{0}$. For the corresponding sequence $\left(\varepsilon_{g}\right)_{n}=\left(\gamma_{g}\right)_{n} \phi$ on $\Lambda_{g n}$ positive lower bounds on modulus exist and are no less than $\zeta_{0_{\Lambda_{g n}}} \inf \mid$.

Under $6\left(c^{\prime}\right)$ consider the sequence of deterministic functions $\left(\varepsilon_{g}\right)_{n}=$ $\left(\gamma_{g}\right)_{n} \phi$ and the difference $\left(\varepsilon_{L 1}\right)_{n}=\varepsilon_{1}-\left(\varepsilon_{g}\right)_{n}$. This is a sequence of piece-wise deterministic continuous functions that converges in $S^{*}$ to the continuous function $\varepsilon_{1 L 1}=\gamma_{L 1} \phi$. Then on a compact set $\Lambda$ for $\zeta_{1} \leq \min \left(\inf _{\Lambda}\left|\varepsilon_{1 L 1}\right|, \zeta_{0_{\Lambda_{g n}}} \inf |\phi|\right)$ we can find the corresponding $N$ so that for $\hat{\varepsilon}_{1 n}=\hat{\varepsilon}_{1 n}-\left(\varepsilon_{g}\right)_{n}+\left(\varepsilon_{g}\right)_{n}$

$$
\operatorname{Pr}\left(\sup \left|\hat{\varepsilon}_{1 n}-\left(\varepsilon_{g}\right)_{n}-\varepsilon_{1 L 1}\right|<\zeta_{1}\right)<\zeta_{2}
$$


Then

$$
\operatorname{Pr}\left(\inf _{A}\left|\hat{\varepsilon}_{1 n}\right|<\zeta_{1}\right)<\operatorname{Pr}\left(\inf _{A}\left|\hat{\varepsilon}_{1 n}-\left(\varepsilon_{g}\right)_{n}\right|<\zeta_{1}\right)<\zeta_{2}
$$

and (23) also holds.

Then with probability approaching 1 on compact $\Lambda$ the continuous random functions $\hat{\varkappa}_{k n}=\hat{\varepsilon}_{1 n}^{-1}\left(\left(\hat{\varepsilon}_{1 n}\right)_{k}^{\prime}-i \hat{\varepsilon}_{2 k n}\right), k=1, \ldots, d$ are in a bounded set in $S^{*}$, and thus $\hat{\phi}_{n}^{-1}(s)=\exp \left(-\int_{0}^{s} \sum_{k=1}^{d} \hat{\varkappa}_{k}(t) d t_{k}\right)$ is also in a bounded set, and thus with probability approaching 1 satisfies the condition for well-posedness of Theorem 5. Then the estimators $\hat{\gamma}_{n}=\hat{\phi}_{n}^{-1} \varepsilon_{1}$ are consistent for $\gamma$ in $S^{*}$ on the compact set $\Lambda$ where $\inf _{\Lambda}\left|\varepsilon_{1 L 1}\right|>0$.

Since $\gamma_{L 1}$ is a continuous function its support is an open set. By Assumption 6(c') the compact support of $\gamma_{g}$ is contained inside the open support of $\gamma_{L 1}$. If $\inf _{\operatorname{supp}\left(\gamma_{l 1}\right)}\left|\varepsilon_{1 L 1}\right|>\zeta_{1}>0$ this concludes the proof of (i).

Step 2. Otherwise consider the set $\bar{\Lambda}=\cup \Lambda_{g n}$; and the open set $\Omega=\operatorname{supp}(\gamma) \backslash \bar{\Lambda}$; then $\hat{\varepsilon}_{1 n}$ converge to $\varepsilon_{1}$ that is continuous on that set. Of course under Assumption $6(\mathrm{c}) \Omega=\operatorname{supp}(\gamma)$. Generally $\Omega$ is a union of open connected sets and we can proceed by considering each component in $\Omega$. Without loss of generality such a component can be assumed to be a connected open set containing zero as an interior point, since if it does not contain zero by a shift (which is a continuous operation in $S^{*}$ ) of an arbitrary interior point into zero this can be attained.

All the proofs that follow apply to an open set that will be denoted $\Omega$ 
that is connected and contains zero as an interior point.

Consider a compact set $\Lambda \subset \Omega$ where $\inf _{\Lambda}\left|\varepsilon_{1}\right|>0$. It follows from the proof in step 1 under continuity of $\varepsilon_{1}$ that $\operatorname{Pr}\left(\sup _{\Lambda}\left|\hat{\varepsilon}_{1 n}^{-1}-\varepsilon_{1}^{-1}\right|>\zeta_{1}\right)=$

$$
\begin{aligned}
& \operatorname{Pr}\left(\sup _{\Lambda} \mid\left(\hat{\varepsilon}_{1 n}^{-1}\left(\varepsilon_{1}-\hat{\varepsilon}_{1 n}\right) \varepsilon_{1}^{-1} \mid>\zeta_{1}\right)\right. \\
\leq & \operatorname{Pr}\left(\sup _{\Lambda} \mid\left(\hat{\varepsilon}_{1 n}^{-1}\left(\varepsilon_{1}-\hat{\varepsilon}_{1 n}\right) \mid>\zeta_{1}^{2}\right)\right. \\
\leq & \operatorname{Pr}\left(\sup _{\Lambda}\left|\hat{\varepsilon}_{1 n}^{-1}\right|>\zeta_{1}^{-1}, \sup _{\Lambda}\left|\hat{\varepsilon}_{1 n}^{-1}\left(\varepsilon_{1}-\hat{\varepsilon}_{1 n}\right)\right|>\zeta_{1}^{2}\right) \\
& +\operatorname{Pr}\left(\sup _{\Lambda}\left|\hat{\varepsilon}_{1 n}^{-1}\right| \leq \zeta_{1}^{-1}, \sup _{\Lambda}\left|\hat{\varepsilon}_{1 n}^{-1}\left(\varepsilon_{1}-\hat{\varepsilon}_{1 n}\right)\right|>\zeta_{1}^{2}\right) \\
\leq & \operatorname{Pr}\left(\sup _{\Lambda}\left|\hat{\varepsilon}_{1 n}^{-1}\right|>\zeta_{1}^{-1}\right)+\operatorname{Pr}\left(\sup _{\Lambda}\left|\varepsilon_{1}-\hat{\varepsilon}_{1 n}\right|>\zeta_{1}^{3}\right) \\
\leq & 2 \zeta_{2} .
\end{aligned}
$$

Theorem 3(b) implies that $\phi^{-1}(s)=\exp \left(-\int_{0}^{s} \sum_{k=1}^{d} \varkappa_{k}(t) d t_{k}\right)$, where $\varkappa_{k}$ is the unique continuous function that solves $\varepsilon_{1} \varkappa-\left(\left(\varepsilon_{1}\right)_{k}^{\prime}-i \varepsilon_{2 k}\right)=0$ in $S^{*}$ on $\Omega$. By Assumptions 6(a-b) for $\phi, \varepsilon_{1} \phi^{-1}$ exists in $S^{*}$ and $g=F t^{-1}\left(\varepsilon_{1} \phi^{-1}\right)$ in $S^{*}$. By Assumption $5 \mathrm{a} \varepsilon_{1}=\gamma \phi$ is non-zero on $\operatorname{supp}(\gamma)$.

Consider the estimator function $\hat{\varkappa}_{k n}=\hat{\varepsilon}_{1 n}^{-1}\left(\left(\hat{\varepsilon}_{1 n}\right)_{k}^{\prime}-i \hat{\varepsilon}_{2 k n}\right)$ on compact $\Lambda \subset \Omega$; the function $\varepsilon_{1}$ is continuous there and it follows that $\left(\varepsilon_{1}\right)_{k}^{\prime}-i \varepsilon_{2 k}=$ $\varepsilon_{1} \varkappa_{k}$ is continuous.

The sequences of random functions $\left(\left(\hat{\varepsilon}_{1 n}\right)_{k}^{\prime}-i \hat{\varepsilon}_{2 k n}\right)$ converge in probability uniformly on $\Lambda$ in $S^{*}$ to the continuous function $\left(\left(\varepsilon_{1}\right)_{k}^{\prime}-i \varepsilon_{k 2}\right)$. Define $\bar{B}=$ 


$$
\begin{aligned}
& \sup _{\Lambda, k}\left|\left(\varepsilon_{1}\right)_{k}^{\prime}-i \varepsilon_{k 2}\right| . \text { For } 0<\zeta_{4} \text { find } N_{2} \text { such that } \\
& \quad \operatorname{Pr}\left(\sup _{\Lambda}\left|\left(\left(\hat{\varepsilon}_{1 n}\right)_{k}^{\prime}-i \hat{\varepsilon}_{2 k n}\right)-\left(\left(\varepsilon_{1}\right)_{k}^{\prime}-i \varepsilon_{k 2}\right)\right|>\zeta_{4}\right)<\zeta_{2} \text { for } n>N_{2} .
\end{aligned}
$$

Bound $\operatorname{Pr}\left(\sup _{\Lambda}\left|\hat{\varkappa}_{k n}-\varkappa_{k}\right|>\zeta_{5}\right) \leq$

$$
\operatorname{Pr}\left(\sup _{\Lambda}\left|\hat{\varepsilon}_{1 n}^{-1}\right| \mid\left(\left(\hat{\varepsilon}_{1 n}\right)_{k}^{\prime}-i \hat{\varepsilon}_{2 n}-\left(\varepsilon_{1}\right)_{k}^{\prime}+i \varepsilon_{2}\left|+\sup _{\Lambda}\right| \hat{\varepsilon}_{1 n}^{-1}-\varepsilon_{1}^{-1}||\left(\varepsilon_{1}\right)_{k}^{\prime}-i \varepsilon_{k 2} \mid>\zeta_{5}\right) \leq\right.
$$

$$
\begin{aligned}
\operatorname{Pr}\left(\sup _{\Lambda}\left|\hat{\varepsilon}_{1 n}^{-1}\right|\right. & \left.>\zeta_{1}^{-1}\right)+\operatorname{Pr}\left(\sup _{\Lambda} \mid\left(\left(\hat{\varepsilon}_{1 n}\right)_{k}^{\prime}-i \hat{\varepsilon}_{2 k n}-\left(\varepsilon_{1}\right)_{k}^{\prime}+i \varepsilon_{k 2} \mid>\zeta_{5} \zeta_{1}\right)\right. \\
+\operatorname{Pr}\left(\sup _{\Lambda}\left|\hat{\varepsilon}_{1 n}^{-1}-\varepsilon_{1}^{-1}\right|\right. & \left.>\zeta_{5} / \bar{B}\right) .
\end{aligned}
$$

If $\zeta_{5}=\min \left\{\zeta_{1} \bar{B}, \zeta_{4} / \zeta_{1}\right\}$ the probability as $n>\max \left\{N_{1}, N_{2}\right\}$ is less than $4 \zeta_{2}$.

$$
\text { Then } \operatorname{Pr}\left(\sup _{\Lambda}\left|\int_{0}^{s} \sum_{k=1}^{d} \hat{\varkappa}_{k n}(t) d t_{k}-\int_{0}^{s} \sum_{k=1}^{d} \varkappa_{k}(t) d t_{k}\right|>\zeta_{6}\right) \leq
$$

$$
\operatorname{Pr}\left(\sup _{\Lambda} \int_{0}^{s} \sum_{k=1}^{d}\left|\hat{\varkappa}_{k n}(t)-\varkappa_{k}(t)\right| d t_{k}>\zeta_{6}\right) \leq \operatorname{Pr}\left(\sup _{\Lambda}\left|\hat{\varkappa}_{k n}(t)-\varkappa_{k}(t)\right|>\zeta_{6} / \mu(\Lambda)\right)
$$

where $\mu(\Lambda)$ is the measure of the compact set $\Lambda$. For $\zeta_{6}=\mu(\Lambda) \zeta_{5}$ then the probability is less than $4 \zeta_{2}$.

Consider now on $\Lambda$ the function $\hat{\phi}_{n}^{-1}(s)=\exp \left(-\int_{0}^{s} \sum_{k=1}^{d} \hat{\varkappa}_{k n}(t) d t_{k}\right)$. Define $\tilde{B}=\sup _{\Lambda} V\left(1+s^{2}\right)^{m} ;$ then $\sup _{\Lambda}\left|\phi^{-1}(s)\right|<\tilde{B}$. 


$$
\begin{aligned}
& \operatorname{Then} \operatorname{Pr}\left(\sup _{\Lambda}\left|\hat{\phi}_{n}^{-1}-\phi^{-1}\right|>\zeta_{7}\right) \leq \\
& \left.\operatorname{Pr} \sup _{\Lambda}\left|\int_{0}^{s} \sum_{k=1}^{d} \hat{\varkappa}_{k n}(t) d t_{k}-\int_{0}^{s} \sum_{k=1}^{d} \varkappa_{k}(t) d t_{k}\right|>\ln \left(1+\tilde{B}^{-1} \zeta_{7}\right)\right),
\end{aligned}
$$

and is smaller than $4 \zeta_{2}$ for $\zeta_{7}=\ln \left(1+\tilde{B}^{-1} \zeta_{6}\right)$.

Since for the continuous functions $\sup _{\Lambda}\left|\phi_{n}^{-1} \hat{\varepsilon}_{1 n}-\phi^{-1} \varepsilon_{1}\right|<$

$$
\tilde{B} \sup _{\Lambda}\left|\varepsilon_{1}-\hat{\varepsilon}_{1 n}\right|+\sup _{\Lambda}\left|\varepsilon_{1}\right| \sup _{\Lambda}\left|\phi_{n}^{-1}-\phi^{-1}\right|
$$

by similar derivations

$$
\operatorname{Pr}\left(\sup _{\Lambda}\left|\hat{\phi}_{n}^{-1} \hat{\varepsilon}_{1 n}-\phi^{-1} \varepsilon_{1}\right|>\zeta_{8}\right)<5 \zeta_{2}
$$

if $\zeta_{8}<\min \left\{\tilde{B}^{-1} \zeta_{1}, \zeta_{7}\left(\sup _{\Lambda}\left|\varepsilon_{1}\right|\right)^{-1}\right.$

If $\Omega$ is bounded then by Assumption $5 \sup _{\Omega}\left|\phi^{-1}(s)\right|$ is uniformly bounded and since $\hat{\phi}_{n}^{-1}$ converges to $\phi^{-1}$ in probability uniformly on any compact set inside $\Omega$ then also $\hat{\phi}_{n}^{-1}$ is bounded away from zero and then $\left|\hat{\phi}_{n}^{-1} \hat{\varepsilon}_{1 n}-\phi^{-1} \varepsilon_{1}\right|$ converges in probability to zero on $\Omega$. This concludes the proof of (i).

Step 3. If $\operatorname{supp}(\gamma)$ is unbounded consider $\tilde{\phi}_{n}^{-1}$ defined in (ii). From the proof in step 1 it follows that for large enough $N$ the estimator $\tilde{\phi}_{n}^{-1}=\hat{\phi}_{n}^{-1}$ on any compact $\Lambda$ with arbitrarily high probability and then $\hat{\varepsilon}_{1 n} \tilde{\phi}_{n}^{-1}$ converges to $\gamma$ on $\Lambda$ in probability in $S^{*}$.

Consider an arbitrary set $\psi_{1}, \ldots, \psi_{v} \in S$ and the corresponding compact 
set $\Lambda$ defined by Lemma 3 and show that $\operatorname{Pr}\left(\left|\left(\tilde{\gamma}_{n}-\gamma, \tilde{\psi}\right)\right|>\zeta\right)$ goes to zero.

Since $\hat{\varepsilon}_{1 n}-\varepsilon_{1}$ converges to zero in probability in $S^{*}$ by Lemma 3 and since on $\Omega$ this difference is a continuous function, then also $\left|\hat{\varepsilon}_{1 n}(t)-\varepsilon_{1}(t)\right|$ converges to zero in probability in $S^{*}$ on $\Omega$. Thus

$$
\int_{\Omega \backslash \Lambda} V\left(1+t^{2}\right)^{m}\left|\hat{\varepsilon}_{1 n}(t)-\varepsilon_{1}(t)\right||\tilde{\psi}(t)| d t
$$

converges in probability to zero. Then since

$$
\begin{aligned}
& \left|\left(\tilde{\gamma}_{n}-\gamma, \tilde{\psi}\right)\right| \leq \sup _{\Lambda}\left|\tilde{\phi}_{n}^{-1} \hat{\varepsilon}_{1 n}-\phi^{-1} \varepsilon_{1}\right| \int_{\Lambda}|\tilde{\psi}(t)| d t \\
& \quad+\int_{\Omega \backslash \Lambda} V\left(1+t^{2}\right)^{m}\left|\varepsilon_{1}(t)\right||\tilde{\psi}(t)| d t \\
& \quad+\int_{\Omega \backslash \Lambda} V\left(1+t^{2}\right)^{m}\left|\hat{\varepsilon}_{1 n}(t)-\varepsilon_{1}(t)\right||\tilde{\psi}(t)| d t
\end{aligned}
$$

it follows that $\operatorname{Pr}\left(\left|\left(\tilde{\gamma}_{n}-\gamma, \tilde{\psi}\right)\right|>\zeta\right) \leq$

$$
\begin{aligned}
\operatorname{Pr}\left(\sup _{\Lambda}\left|\hat{\phi}_{n}^{-1} \hat{\varepsilon}_{1 n}-\phi^{-1} \varepsilon_{1}\right|\right. & \left.>\left(\int_{\Lambda}|\tilde{\psi}(t)| d t\right)^{-1} \zeta\right) \\
+\operatorname{Pr}\left(\int_{\Omega \backslash \Lambda} V\left(1+t^{2}\right)^{m}\left|\varepsilon_{1}(t)\right||\tilde{\psi}(t)| d t\right) & >\zeta) \\
+\operatorname{Pr}\left(\int_{\Omega \backslash \Lambda} V\left(1+t^{2}\right)^{m}\left|\hat{\varepsilon}_{1 n}(t)-\varepsilon_{1}(t)\right||\tilde{\psi}(t)| d t\right) & >\zeta)
\end{aligned}
$$

Here as shown in the Step 2 the first probability converges to zero, the second converges to zero by assumption 6 on $\varepsilon_{1}$, definition of the set $\Lambda$ 
and Lemma 3 , and the third by convergence of $\hat{\varepsilon}_{1 n}$. Then $\tilde{\gamma}_{n}$ converges in probability to $\gamma=\phi^{-1} \varepsilon_{1}$ in $S^{*}$. Taking inverse Fourier transforms in $S^{*}$ concludes the proof.

The Theorem provides consistency of plug-in estimators for solutions to the system of equations (16) and consequently (3) in a fairly general set-up. Nevertheless some assumptions can be further relaxed. Of course, establishing results for a compact support of the Fourier transforms is much easier and thus using spectral cut-off can be advantageous especially when high frequency components of the regression function may be commesurate with the magnitude of the error components.

Computation of the estimators requires applying Fourier transforms and inverse Fourier transforms. This can be accomplished with numerical algorithms. However, it is possible to simplify the estimated $\hat{\varepsilon}$. Consider instead of the estimator in (22), $\hat{w}_{1}(z)_{n}$, an estimator computed as $\left.\sum_{i=1}^{n} \alpha_{i}^{-1} y_{i} K \frac{z_{i}-z}{h}\right)$ with the weight $\alpha_{i}=\sum_{j \neq i}^{n} K\left(\frac{z_{j}-z_{i}}{h}\right)$ replacing the $z$-dependent weight in $\hat{w}_{1}(z)_{n}$ and similar estimators for $\hat{w}_{2 k}(z)_{n}$. Then the corresponding Fourier transform $\hat{\varepsilon}_{1 n}(s)$ can be expressed as $\sum_{i=1}^{n} \alpha_{i}^{-1} y_{i} e^{i s^{T} z_{j}} \operatorname{sinc}\left(\frac{s^{T} z_{j}}{\pi}\right)$, where by definition $\operatorname{sinc}(x)=\frac{\sin \pi x}{\pi x}$, and similar expressions for $\hat{\varepsilon}_{2 k n}$. Further computation for the estimators would have to be done numerically. 


\section{Conclusion}

This paper was devoted to treating a single convolution equation and a specific system of convolution equations; many statistical models with various independence conditions give rise to such equations; measurement error is emphasized here, but equations of this type are also applicable in other models, such as factor models and panel data models; many examples are presented in Zinde-Walsh (2012). The results of this paper indicate conditions for identification and well-posedness when casting these equations in terms of generalized functions; the generalized functions approach enlarges the area of applicability of the models.

\section{References}

[1] An, Y, and Y. Hu (2012) Well-posedness of measurement error models for self-reported data, Journal of Econometrics, vol. 168 (2012), pp. 259269.

[2] Antosik, P., J.Mikusinski and R. Sikorski, (1973) Theory of Distributions. The Sequential approach. Elsevier-PWN, Amsterdam-Warszwa.

[3] Carroll, R. J., Ruppert, D., Stefanski, L. A. and C.M. Crainiceanu (2006). Measurement Error in Nonlinear Models: A Modern Perspective. Chapman \& Hall. 
[4] Carrasco, M., J.-P. Florens, and E. Renault (2007) Linear Inverse Problems in Structural Econometrics Estimation Based on Spectral Decomposition and Regularization, Handbook of Econometrics, Vol. 6B, ed. by J.Heckman and E. Leamer. Amsterdam: North-Holland.

[5] Carrasco, M. and J.-P. Florens (2010), A Spectral Method for Deconvolving a Density, Econometric Theory, 27 , pp 546-581.

[6] Chen, X., H. Hong and D.Nekipelov (2011), Nonlinear models of measurement errors, Journal of Economic Literature, 49, pp.901-937.

[7] Cohen, A.C. Jr (1951) Estimating the Mean and Variance of Normal Populations from Singly Truncated and Doubly Truncated Samples, Annals of Mathematical Statistics, 21(4), pp. 557-569.

[8] Cunha, F., J.J. Heckman and S.M.Schennach (2010), Estimating the Technology of Cognitive and Noncognitive Skill Formation, Econometrica, v.78, 883-933.

[9] Devroye, L. (1978), The Uniform Convergence of the Nadaraya-Watson Regression Function Estimate, The Canadian Journal of Statistics, 6, pp.179-191.

[10] Devroye, L. and L. Győrfi (1985) Nonparametric Density Estimation: The $L_{1}$ View, Wiley, New York.

[11] Fan, J. Q. (1991), On the Optimal Rates of Convergence for Nonparametric Deconvolution Problems," Annals of Statistics, 19, 1257-1272. 
[12] Gel'fand, I.M. and G.E.Shilov (1964) Generalized Functions, Vol.1, Properties and Operations, Academic Press, San Diego.

[13] Gel'fand, I.M. and G.E.Shilov (1964) Generalized Functions, Vol.2, Spaces of Test functions and Generalized Functions, Academic Press, San Diego.

[14] Gel'fand, I.M. and N.Ya Vilenkin (1964) Generalized Functions, Vol.4, Applications of Harmonic Analysis, Academic Press, San Diego.

[15] Hadamard, J. (1923): Lectures on Cauchy's Problem in Linear Partial Differential Equations. Yale University Press, New Heaven.

[16] Hall, P. and A.Yatchew (2007) Nonparametric Estimation When Data on Derivatives are Available, Annals of Statistics, 35:1, 300-323.

[17] Hausman, J. A., Newey, W. K., Ichimura, H. and J. Powell (1991) Identification and estimation of polynomial errors-in-variables models, Journal of Econometrics, 50:3, 273-295.

[18] Hirata, Y. and H. Ogata (1958), On the exchange formula for distributions, J.Sci. Hiroshima Univ. Ser.A, 22, pp.147-152.

[19] Hu, Y. (2008), Identification and Estimation of Nonlinear Models with Misclassification Error using Instrumental Variables: A General Solution, Journal of Econometrics, 144, pp. 27-61. 
[20] Kaminski, A (1982), Convolution, product and Fourier transform of distributions, Studia Mathematica, v. 74, pp.83-96.

[21] Kaminski, A and R. Rudnicki (1991), A note on the convolution and the product $D^{\prime}$ and $S^{\prime}$, Internat. J. Math\&Math Sci., v.14, 275-282.

[22] Kirillov, A.A. and A.D.Gvishiani (1982), Theorems and Problems in Functional Analysis, Springer-Verlag.

[23] Klann E., M.Kuhn, D.A.Lorenz, P.Maass and H.Thiele (2007) Srinkage versus deconvolution, Inverse problems, 23, pp.2231-2248.

[24] Koralov, L. and Ya.Sinai (2007), Theory of Probability and Random Processes, Springer.

[25] Kotlyarski, I. (1967), On Characterizing the Gamma and Normal Distribution, Pacific Journal of Mathematics, 20, pp. 69-76.

[26] A.Meister (2009) Deconvolution problems in nonparametric statistics, Lecture notes in statistics, Springer-Verlag.

[27] Newey, W. (2001), Flexible Simulated Moment Estimation of Nonlinear Errors-in-Variables Models, Review of Economics and Statistics, v. 83, 616-627.

[28] Schwartz, L. (1966) "Théorie des distributions", Hermann, Paris.

[29] Schennach, S. (2007) Instrumental variable estimation in nonlinear errors-in-variables models, Econometrica, v.75, pp. 201-239. 
[30] Stone, Ch.J. (1982) Optimal global rates of convergence for nonparametric regression, The Annals of Statistics, v. 10, 1040-1053.

[31] Sobolev, S.L. (1992) Cubature Formulas and Modern Analysis. Gordon and Breach Science Publishers.

[32] Wang, L. and Ch.Hsiao (2011), Method of Moments estimation and identifiability of semiparametic nonlinear errors-in-variables models, Journal of Econometrics, pp. 901-937.

[33] Zinde-Walsh, V. (2008), Kernel Estimation when Density May not Exist, Econometric Theory, v.24, 696-725.

[34] Zinde-Walsh, V. (2009), Errors-in-variables models: a generalized functions approach, working paper, arXiv:0909.5390v1 [stat.ME], McGill University working paper.

[35] Zinde-Walsh, V. (2011), Presidential Address: Mathematics in economics and econometrics, Canadian Journal of Economics, v.44, pp. 1052-1068.

[36] Zinde-Walsh (2012) Identification and well-posedness in nonparametric models with independence conditions, submitted chapter, Handbook of Applied Nonparametric and Semiparametric Econometrics and Statistics, Oxford University Press. 